\title{
Akt-mediated phosphorylation of Bmi1 modulates its oncogenic potential, E3 ligase activity, and DNA damage repair activity in mouse prostate cancer
}

\author{
Karim Nacerddine, ${ }^{1}$ Jean-Bernard Beaudry, ${ }^{1}$ Vasudeva Ginjala, ${ }^{2}$ Bart Westerman, ${ }^{1}$ \\ Francesca Mattiroli, ${ }^{3}$ Ji-Ying Song, ${ }^{4}$ Henk van der Poel, ${ }^{5}$ Olga Balagué Ponz, ${ }^{6}$ \\ Colin Pritchard, ${ }^{1}$ Paulien Cornelissen-Steijger, ${ }^{1}$ John Zevenhoven, ${ }^{1}$ Ellen Tanger, ${ }^{1}$ \\ Titia K. Sixma, ${ }^{3}$ Shridar Ganesan, ${ }^{2}$ and Maarten van Lohuizen ${ }^{1}$

\begin{abstract}
1Division of Molecular Genetics and Center for Biomedical Genetics, The Netherlands Cancer Institute, Amsterdam, The Netherlands. ${ }^{2}$ Cancer Institute of New Jersey, Robert Wood Johnson Medical School-UMDNJ, New Brunswick, New Jersey, USA. ${ }^{3}$ Division of Biochemistry and Center for Biomedical Genetics, ${ }^{4}$ Division of Experimental Animal Pathology, ${ }^{5}$ Division of Surgical Oncology and ${ }^{6}$ Division of Pathology, The Netherlands Cancer Institute, Amsterdam, The Netherlands.
\end{abstract}

\begin{abstract}
Prostate cancer (PCa) is a major lethal malignancy in men, but the molecular events and their interplay underlying prostate carcinogenesis remain poorly understood. Epigenetic events and the upregulation of polycomb group silencing proteins including Bmi1 have been described to occur during PCa progression. Here, we found that conditional overexpression of $\mathrm{Bmi} 1$ in mice induced prostatic intraepithelial neoplasia, and elicited invasive adenocarcinoma when combined with PTEN haploinsufficiency. In addition, Bmi1 and the PI3K/Akt pathway were coactivated in a substantial fraction of human high-grade tumors. We found that Akt mediated Bmi1 phosphorylation, enhancing its oncogenic potential in an Ink4a/Arf-independent manner. This process also modulated the DNA damage response and affected genomic stability. Together, our findings demonstrate the etiological role of Bmi1 in PCa, unravel an oncogenic collaboration between Bmi1 and the PI3K/Akt pathway, and provide mechanistic insights into the modulation of $\mathrm{Bmi1}$ function by phosphorylation during prostate carcinogenesis.
\end{abstract}

\section{Introduction}

Prostate cancer (PCa) is the most frequently diagnosed noncutaneous malignancy and a leading cause of cancer death among men in the Western world (1). PCa develops in gradual stages that display increasing pathology, ranging from premalignant low- and high-grade prostatic intraepithelial neoplasia (PIN) to capsule-confined and invasive carcinoma, ultimately evolving to lethal hormone-refractory metastatic disease. Despite its clinical impact, the molecular mechanisms underlying prostate carcinogenesis remain poorly understood. Several genetic events have been implicated in PCa development, including alteration of $P T E N, C D K N 1 B$ (also referred to as $p 27$ ), $R B$, androgen receptor, $R N a s e L$, and NKX3.1; amplification of Bcl2 and $c-M y c$ oncogenes; and TMPRSS2-ETS fusion gene rearrangements (2). Among the most frequent, PTEN monoallelic loss is found in $30 \%-70 \%$ of PCa patients at the time of diagnosis, whereas loss of heterozygosity is associated with advanced PCa (3-5). PTEN antagonizes the actions of PI3K by dephosphorylating the lipid second messenger phosphatidylinositol 3,4,5-trisphosphate (PIP3) and thereby exerts its tumor suppressor function mainly by restraining the PI3K/Akt prosurvival pathway (6). There is no evidence of germline PTEN mutations in hereditary prostate cancer $(7,8)$, which suggests that interactions between PTEN haploinsuf-

Authorship note: Karim Nacerddine and Jean-Bernard Beaudry contributed equally to this work.

Conflict of interest: The authors have declared that no conflict of interest exists. Citation for this article: J Clin Invest. 2012;122(5):1920-1932. doi:10.1172/JCI57477. ficiency and other genetic and/or epigenetic determinants are required for prostate tumorigenesis.

In the past years, epigenetic events such as DNA methylation and histone modification have emerged as important mechanisms in the course of $\mathrm{PCa}(9)$. In line with this, upregulation of polycomb group $(\mathrm{PcG})$ epigenetic silencers EZH2, Ring1B, and Bmi1 has been shown to accompany PCa progression and correlate with poor clinical outcome (10-14).

Bmi1 is a core component of the polycomb repressive complex 1 (PRC1). It forms a heterodimer with Ring1B/Rnf2 and stimulates its ubiquitin ligase activity toward lysine 119 of histone $2 \mathrm{~A}$ (H2A-K119) $(15,16)$, thereby contributing to a crucial mechanism of PcG-mediated gene silencing (17). Bmi1 was initially identified as an oncogene cooperating with $c-M y c$ in lymphomagenesis (18); later, Bmil upregulation was detected in a variety of human malignancies, including $\mathrm{PCa}$ (19). Bmi1 protein also undergoes phosphorylation $(20,21)$ at 3 highly conserved serines (22-30), which suggests that, beyond the cellular level, Bmi1 activity may also be regulated posttranslationally. In addition to its role in cellular transformation, Bmi1 is also a critical regulator of cellular senescence and stem cell self-renewal $(31,32)$. Mechanistically, Bmi1 functions are largely attributed to its potent transcriptional repression of the CDKN2A locus that encodes $\mathrm{p} 16^{\mathrm{INK} 4 \mathrm{~A}}$ and $\mathrm{p} 19^{\mathrm{ARF}} /$ p14 ARF cell cycle regulators $(31,33)$, although Ink4a/Arf-independent functions of Bmi1 have also been demonstrated $(34,35)$.

Despite correlative evidence, a causal role for Bmi1 in PCa, alone or in the context of additional somatic mutations, has not been demonstrated. In the current study, we generated a 


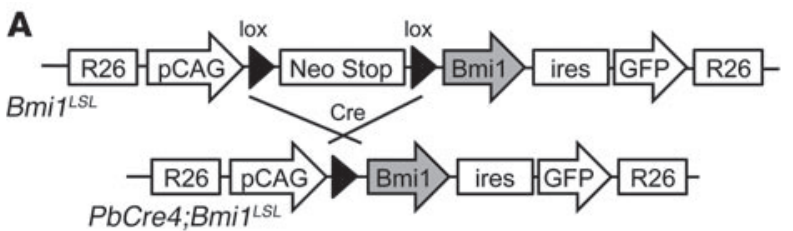

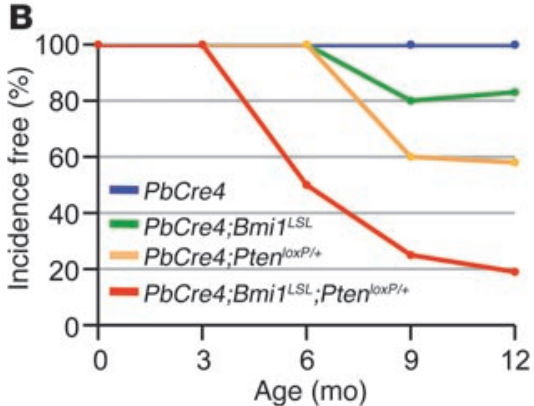

Age (mo)
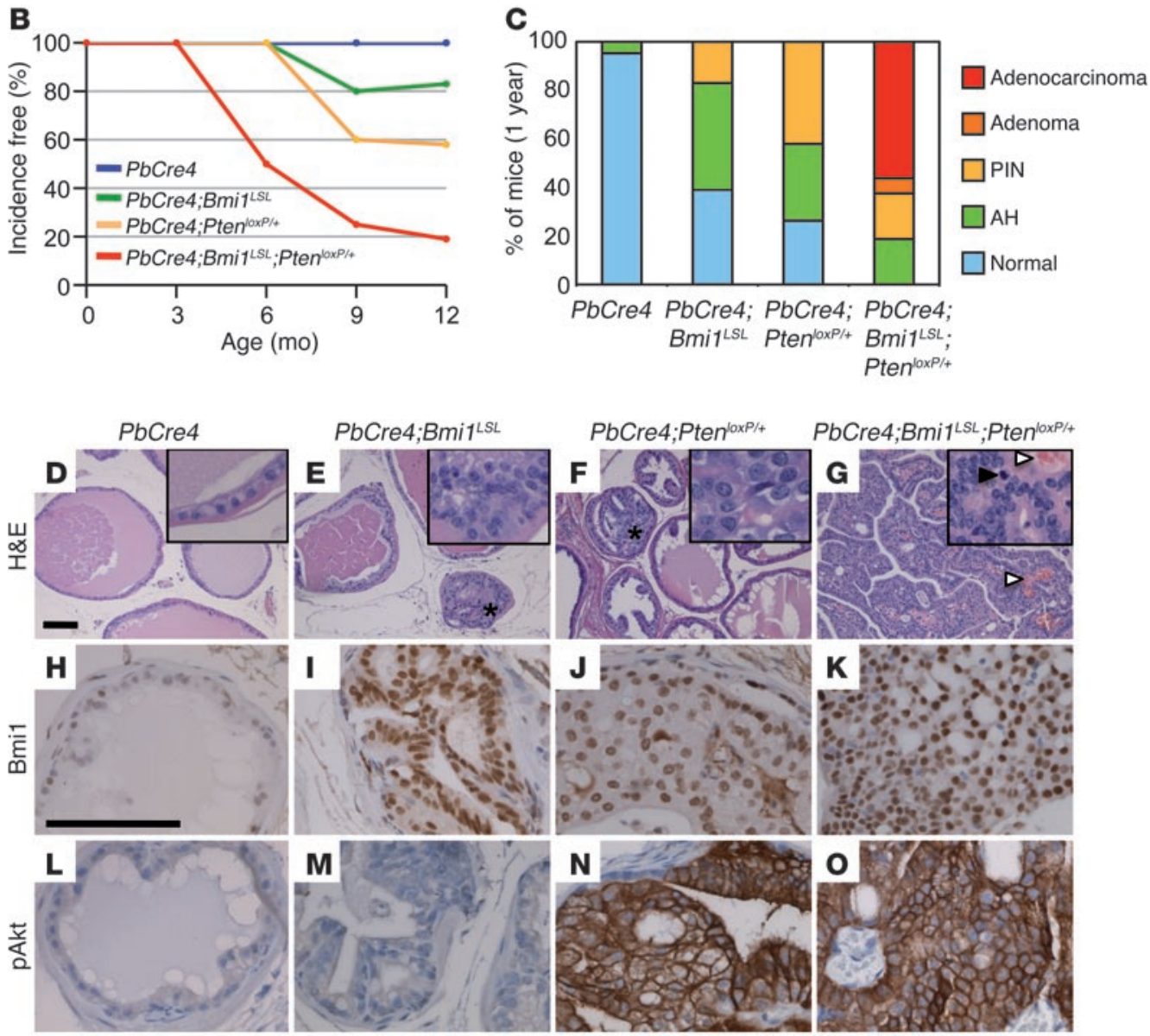

Figure 1

Bmi1 cooperates with PTEN haploinsufficiency in prostate cancer progression. (A) Schematic representation of the ROSA26 Lox-STOP-Lox Bmi1 allele (Bmi1LSL). (B) PIN- and carcinoma-free mice at the indicated ages. $n=29$ (PbCre4); 36 (PbCre4;Bmi1 $\left.{ }^{L S L}\right) ; 26$ (PbCre4;Pten $\left.{ }^{l o x P /+}\right) ; 24$ (PbCre4;Bmi1LSL;Pten $\left.{ }^{\text {loxP } /+}\right)$. (C) Prostate histopathology of 1-year-old animals. PbCre4 controls $(n=21)$ appeared mostly normal. PbCre4;Bmi1 1 LSL $(n=25)$ and $P b C r e 4 ; P t e n^{10 x P /+}(n=19)$ mice developed atypical hyperplasia $(\mathrm{AH})$ and PIN lesions with incomplete penetrance, whereas only PbCre4;Bmi1 LSL;Pten ${ }^{\text {IoxP/+ }}$ mice $(n=16)$ developed prostate adenocarcinoma. (D-G) H\&E-stained prostate sections. (D) Normal epithelium in PbCre4 controls. (E) PIN lesion (asterisk) in PbCre4;Bmi1LSL mice. (F) PIN lesion (asterisk) in PbCre4;Pten ${ }^{/ o x P /+}$ mice. (G) Adenocarcinoma

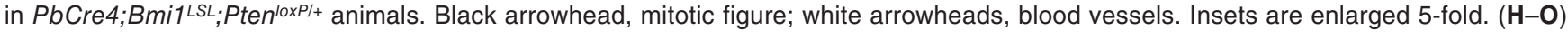
Immunohistochemical expression analysis of Bmi1-phosphorylated Akt (H-K) and pAkt (S473) (L-O). Scale bars: $100 \mu \mathrm{m}$.

conditional transgenic mouse model in order to address the role of forced prostate-specific expression of Bmil in PCa etiology. We showed that the rise of Bmil led to PIN formation and acted synergistically with PTEN haploinsufficiency in the transition from PIN to invasive cancer. We provided evidence that this cooperation occurred through Akt-mediated phosphorylation of Bmi1, a posttranslational modification that increased its oncogenic potential, stimulated H2A-K119 ubiquitination in vitro and at sites of DNA double-strand breaks (DSBs) and promoted homologous recombination (HR) and genomic instability in PCa.

\section{Results}

Overexpression of Bmi1 induces neoplastic transformation of the prostate epithelium. In order to gain more insight into the etiological role of Bmi1 elevation in prostate tumorigenesis, we generated mice with conditional, Cre-mediated Bmi1 overexpression in the prostate epithelium (Figure 1A and Supplemental Methods; supplemental material available online with this article; doi:10.1172/JCI57477DS1). Expression of the ROSA26 Lox-STOP-Lox Bmi1 allele (Bmi1 ${ }^{\text {LSL }}$ transgene) in the luminal cells of the prostate epithelium was achieved by crossing $\mathrm{Bmi1}^{\mathrm{LSL}}$ animals to the ARR2Probasin-Cre ( $\left.\mathrm{PbCre} 4\right)$ transgenic line (ref. 36 and Supplemental Figure 1A). 


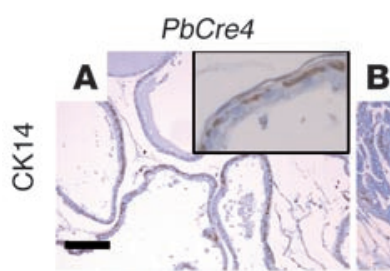

PbCre4;Bmi1 ${ }^{\text {LSL }}$
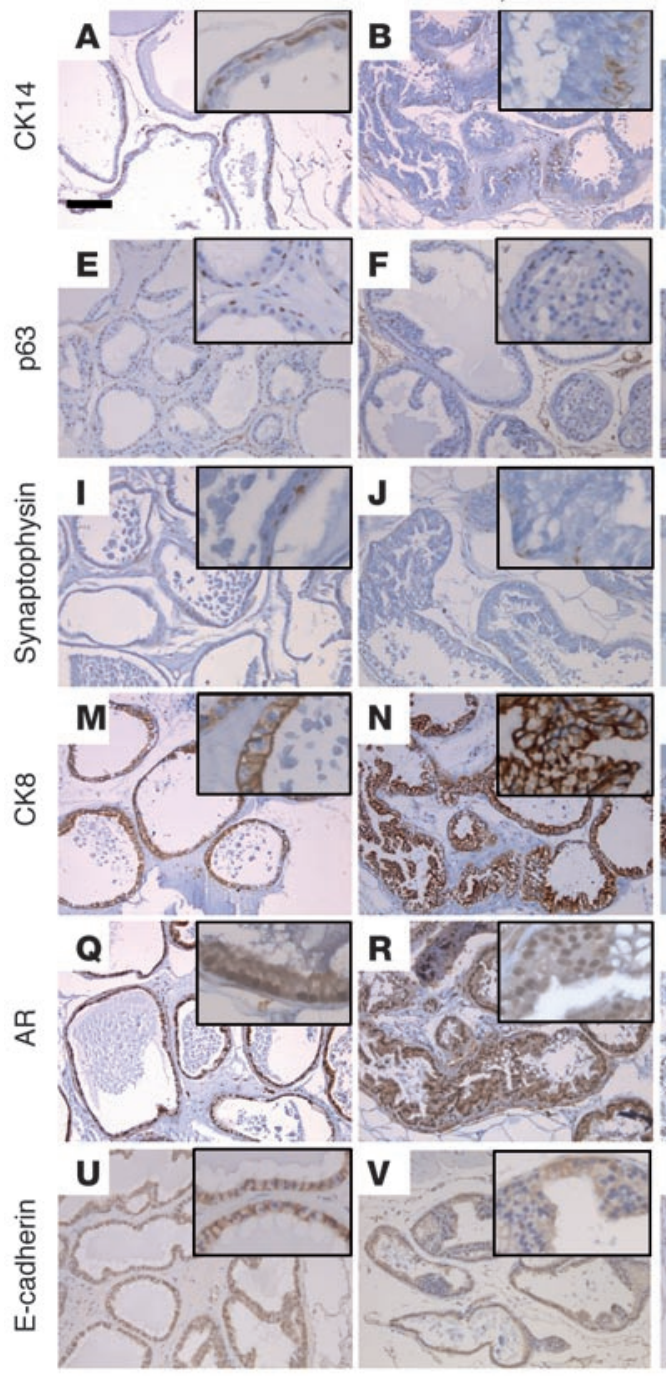

PbCre4;Pten ${ }^{\text {loxp/+ }}$
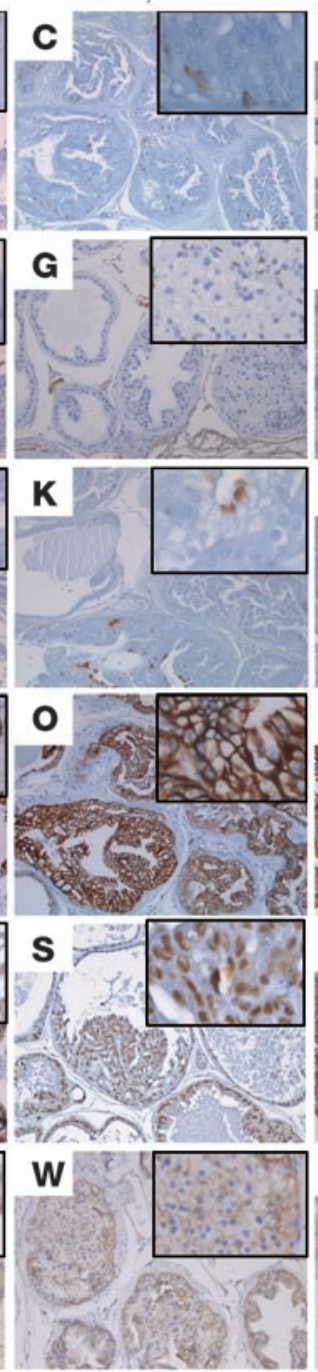

PbCre4;Bmi ${ }^{\text {LSL }} ;$ Pten $^{\text {loxP/+ }}$
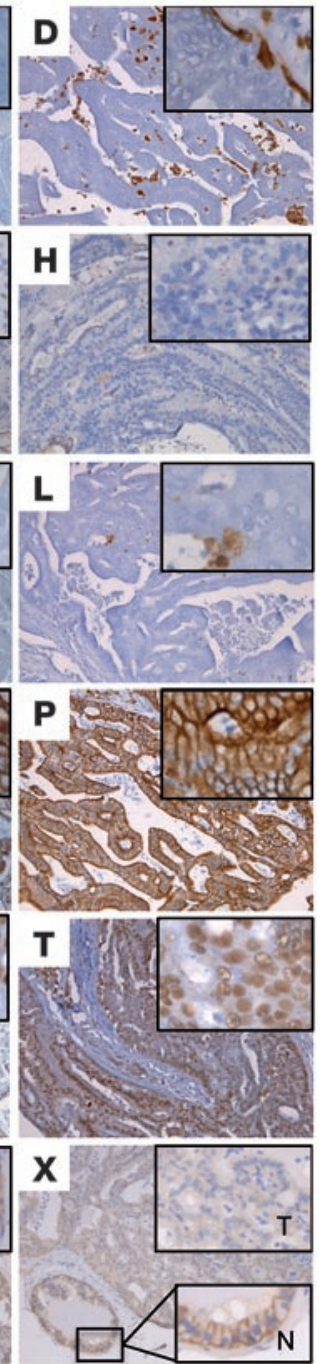

Figure 2

Secretory luminal epithelial phenotype of PbCre4;Bmi1 LSL;Pten ${ }^{\text {loxP/+ }}$ prostate cancer cells. Immunohistochemical staining of formalinfixed paraffin-embedded prostate tissue sections. (A-D) Cytokeratin 14 (CK14) and (E-H) p63, marking basal cells. (I-L) Synaptophysin, marking neuroendocrine cells. (M-P) Cytokeratin 8 (CK8), (Q-T) androgen receptor (AR), and (U-X) E-cadherin, marking secretory luminal cells. Insets are enlarged 5 -fold; in $\mathbf{X}$, insets show both tumor $(\mathrm{T})$ and normal prostatic duct $(\mathrm{N})$. Scale bar: $100 \mu \mathrm{m}$.
Mouse cohorts were systematically analyzed at 3, 6, 9, and 12 months. At 6 months, atypical hyperplastic foci were observed in the prostate lobes of $\mathrm{PbCre} 4 ; \mathrm{Bmi1}{ }^{L S L}$ mice. These early lesions were characterized by enlargement of nuclear diameter, thickening of nuclear membrane, hyperchromasia, and conspicuous presence of nucleoli. From 9 months onward, about $20 \%$ of PbCre 4 ; Bmi $1^{L S L}$ animals showed multifocal PIN lesions, a precursor stage of $\mathrm{PCa}$ (Figure 1, B and C). PbCre4;Bmi1 ${ }^{\text {LSL }}$ PINs displayed micropapillary, cribriform, and/or tuft-like structural organizations reminiscent of human low-grade PINs (Figure 1E and Supplemental Figure 1B). However, histopathological analysis of a large cohort of $\mathrm{PbCre} 4 ; \mathrm{Bmi} 1^{L S L}$ males did not show adenocarcinoma lesions over a year of monitoring. These data demonstrated that Bmi1 overexpression in the prostate was sufficient to initiate epithelial neoplastic transformation.

Bmil cooperates with PTEN haploinsufficiency in prostate cancer progression. The absence of adenocarcinoma in $\mathrm{PbCre} 4$; Bmi $1^{L S L}$ prostates prompted us to investigate whether additional mutations could collaborate with Bmil overexpression to promote tumorigenesis. We addressed this in the context of heterozygous PTEN deletion because (a) PTEN monoallelic mutations are often detect- ed in human primary $\mathrm{PCa}$, (b) Bmi1 exerts a positive effect on Akt activation in breast cancer cells $(37,38)$, and (c) Bmi1 represses PTEN expression in nasopharyngeal cancer cells (39).

At 9 months of age, nearly $40 \%$ of PbCre4;Pten ${ }^{l o x P /+}$ mice developed multifocal PIN lesions that never progressed to carcinoma (Figure 1, B, C, and F, and Supplemental Figure 1B), confirming previous reports (40). Analysis of the compound mutant mice revealed that elevation of $\mathrm{Bmi} 1$ and PTEN heterozygous loss displayed striking cooperativity. Aside from a significantly shortened latency of PIN lesions to 6 months of age, from 9 months, PbCre4; Bmi1 ${ }^{L S L}$;Pten ${ }^{\text {loxP/+ }}$ mice developed prostate adenocarcinomas that reached a penetrance of $55 \%$ at 1 year (Figure 1, B, C, and G, and Supplemental Figure 1B).

PbCre4;Bmi1 ${ }^{\text {LSL; }}$ Pten ${ }^{\text {loxP/+ }}$ tumors were multifocal, with predominant localization in the dorsolateral lobe, accompanied by frequent bladder outlet obstruction, locally invasive and highly vascularized (Supplemental Figure 2, A and B, and Figure 1G). Stromal reaction as well as lymphocyte and neutrophil infiltrations were frequently observed (data not shown). Histologically, adenocarcinomas were composed of poorly differentiated cells with perturbed polarity, as well as areas of picnotic/necrotic cells (Figure 1G). Lesions were 
A

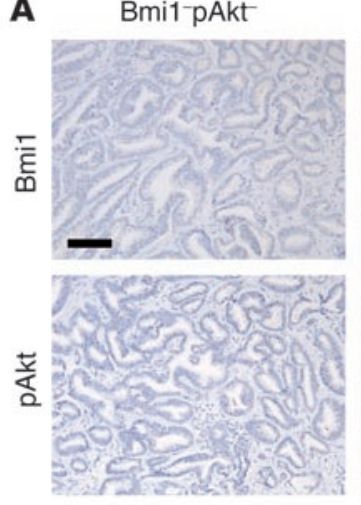

Bmi1 ${ }^{+p A k t}$
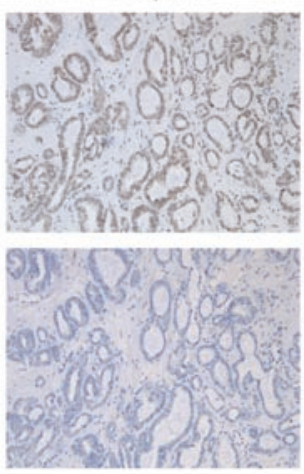
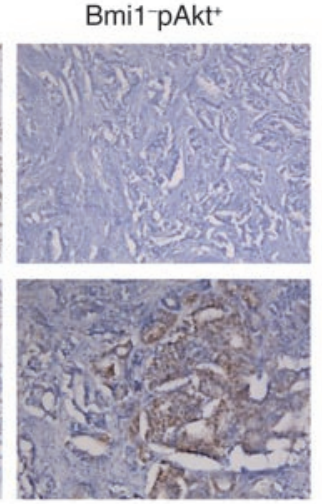

Bmi1 ${ }^{+p A k t}$
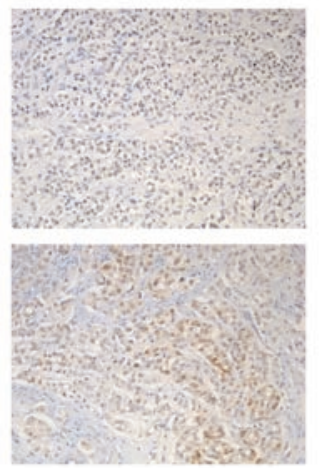

B

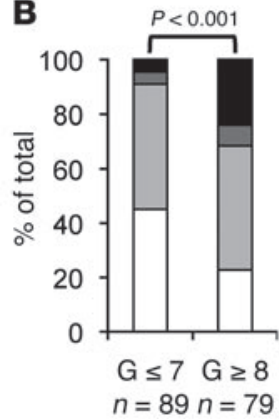

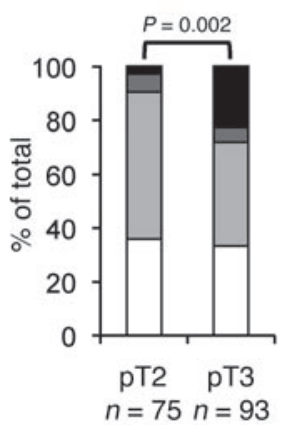

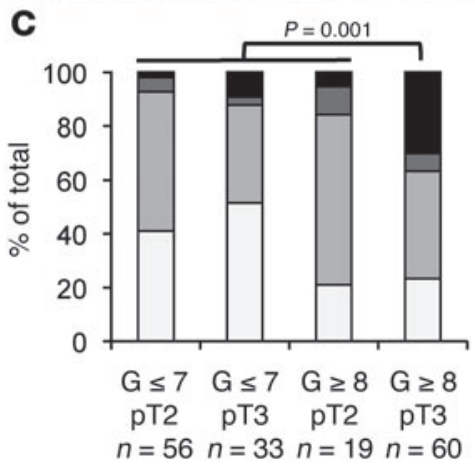

Figure 3

Bmi1 expression and activation of PI3K/Akt pathway correlate in high-grade prostate cancer clinical samples. (A) Representative immunohistochemical staining for Bmi1 and pAkt on adjacent sections of formalinfixed, paraffin-embedded human prostate cancer specimens. Scale bar: $100 \mu \mathrm{m}$. (B) Immunohistochemical assessment of Bmi1 and pAkt expression status in 168 human prostate tumors categorized according to histological or clinical grade (see Table 1). Bmi1+pAkt+ tumors were associated with high Gleason scores $(P<0.001)$ and with advanced pathological stage $(P=0.002)$. (C) Immunohistochemical assessment of Bmi1 and pAkt expression status in 168 human prostate tumors categorized according to both histological and clinical grade (see Table 1). Bmi1 ${ }^{+} p A \mathrm{kt}^{+}$tumors were associated with worsening of both Gleason score and pathological stage (i.e., Gleason $\geq 8$ and $\mathrm{pT} 3 ; P=0.001$ ).

highly proliferative, as indicated by the prevalence of mitotic figures and the abundance of Ki-67-labeled nuclei (Figure 1G and Supplemental Figure 2C). Metastases were not detected over the course of monitoring (lymph nodes, testes, kidney, lung, liver, pelvis, and femur were systematically analyzed).

Prostate immunohistological analysis of the entire animal cohort revealed that $\mathrm{PbCre} 4 ; \mathrm{Bmi1} \mathrm{LSL}^{\mathrm{LS}}$ and $\mathrm{PbCre} 4 ; \mathrm{Bmi1}^{\mathrm{LSL}} ; \mathrm{Pten}^{\text {loxP } /+}$ prostate consistently expressed high levels of Bmi1, whereas PbCre4;Pten ${ }^{\text {loxP/+ }}$ and PbCre 4;Bmi1 ${ }^{\text {LSL }}$;Pten ${ }^{\text {loxP/+ }}$ prostate showed upregulation of phosphorylated Akt (pAkt; Figure 1, H-O, and Supplemental Figures 3 and 4), indicative of PI3K/Akt pathway activation. Together, these results demonstrated the oncogenic collaboration between Bmi1 and PTEN haploinsufficiency and identified a Bmi1-PI3K/ Akt synergistic axis driving invasive prostate carcinogenesis.

PbCre 4;Bmi1 ${ }^{\text {LSL }}$;Pten ${ }^{\text {lox } /{ }^{+}+}$tumors recapitulate features of buman PCa. The prostate epithelium consists of 3 differentiated cell types: luminal-secretory, basal, and neuroendocrine. In order to identify the subpopulation of cells present in the PINs and adenocarcinoma, prostates from different mouse genotypes were analyzed for expression of epithelial markers by immunohistochemistry. PINs arising in $\mathrm{PbCre} 4 ; \mathrm{Bmi1}{ }^{L S L}$ and $\mathrm{PbCre} 4 ; \mathrm{Pten}^{\mathrm{loxP} /+}$, as well as PbCre4; Bmi1 ${ }^{\text {LSL }}$;Pten ${ }^{\text {lox } P /+}$ adenocarcinoma, almost exclusively consisted of secretory luminal epithelial cells positive for cytokeratin 8 and

androgen receptor, whereas basal cells expressing cytokeratin 14 and p63 and neuroendocrine cells expressing synaptophysin were virtually absent (Figure 2, A-T). E-cadherin expression was markedly reduced in $\mathrm{PbCre} 4 ; \mathrm{Bmi1}{ }^{\mathrm{LSL}}$ and PbCre4;Pten ${ }^{\text {loxP/+ }}$ PINs and almost completely suppressed in PbCre4;Bmi1 ${ }^{\text {LSL }} ;$ Pten $^{\text {loxP/+ }}$ tumors (Figure 2, U-X), consistent with the perturbed cell polarity and the invasive trait of these lesions. This cellular phenotype showed striking similarities with the human disease, which has a markedly luminal phenotype with frequent loss of E-cadherin expression, while absence of basal cells is used as a diagnostic criterion. We conclude that PbCre4;Bmil ${ }^{\text {LSL }}$;Pten ${ }^{\text {loxP/+ }}$ compound mice constitute a bona fide model for PCa studies.

Association of Bmil expression and PI3K/Akt activation in bighgrade buman prostate cancer. In order to evaluate the clinical relevance of Bmi1/PI3K/Akt oncogenic synergy, we used immunohistochemistry and conducted Bmi1 and pAkt (S473) expression analyses on a total of 168 paraffin-embedded human prostate tumors (Figure 3A). Clinical tissue specimens were selected from patients subjected to radical prostatectomy at our institution and classified according to their respective histopathological grade (postoperative Gleason score) or clinical stage (TNM system). Histological categories divided tumors with Gleason score of 7 or less from those with Gleason score of 8 or more (Gleason $\leq 7,89$ cases; Gleason $\geq 8 ; 79$ cases), whereas clinical stage groups were defined as prostate-confined disease (pT2, 75 cases) and disease with extraprostatic extension (pT3, 93 cases). The results are summarized in Figure 3, B and C, and detailed in Table 1. The total proportion of Bmil-expressing tumors (i.e., Bmi1 ${ }^{+} \mathrm{pAkt}^{-}$and $\mathrm{Bmi}^{+} \mathrm{pAkt}^{+}$) appeared nearly equal between pT2 and pT3 stages $(57.3 \%$ and $61.3 \%$, respectively) and increased in Gleason $\geq 8$ compared with Gleason $\leq 7$ tumors (69.6\% versus 50.5\%; Figure 3B and Table 1). This is consistent with a previous report suggesting that Bmi1 is overexpressed from early stages of the disease onward (13). pAkt-positive specimens (i.e., Bmi1-pAkt and $\mathrm{Bmi}^{+} \mathrm{pAkt}^{+}$) were predominantly found in the Gleason $\geq 8$ group $(31.6 \%$, versus $9 \%$ in Gleason $\leq 7)$ and within the pT3 grade tumors ( $28 \%$ versus $9.4 \%$ in pT2; Figure 3B and Table 1).

Remarkably, we found a significant correlation between Bmi1 and pAkt coexpression (i.e., $\mathrm{Bmi1}^{+} \mathrm{pAkt}^{+}$) and worsened pathological grade (Gleason $\geq 8,24 \%$; Gleason $\leq 7,4.5 \% ; P<0.001$ ). Similarly, 
Table 1

Association of Bmi1 and pAkt immunoreactivity with high-grade human prostate cancer

$\begin{array}{lccccc}\text { Group } & \begin{array}{c}\text { No. of } \\ \text { specimens }\end{array} & \begin{array}{c}\text { Bmi1-pAkt- } \\ \text { Bmitivanoreactivity }\end{array} \\ \text { Gleason } \leq 7 \text { and pT2 } & n=56 & 23(41 \%) & 29(51.8 \%) & 3(5.4 \%) & 1(1.8 \%) \\ \text { Gleason } \leq 7 \text { and pT3 } & n=33 & 17(51.5 \%) & 12(36.4 \%) & 1(3 \%) & 3(9.1 \%) \\ \text { Gleason } \geq 8 \text { and pT2 } & n=19 & 4(21 \%) & 12(63.2 \%) & 2(10.5 \%) & 1(5.3 \%) \\ \text { Gleason } \geq 8 \text { and pT3 } & n=60 & 14(23.3 \%) & 24(40 \%) & 4(6.7 \%) & 18(30 \%) \\ \text { All Gleason } \leq 7 & n=89 & 40(45 \%) & 41(46 \%) & 4(4.5 \%) & 4(4.5 \%) \\ \text { All Gleason } \geq 8 & n=79 & 18(22.8 \%) & 36(45.6 \%) & 6(7.6 \%) & 19(24 \%) \\ \text { All pT2 } & n=75 & 27(36 \%) & 41(54.6 \%) & 5(6.7 \%) & 2(2.7 \%) \\ \text { All pT3 } & n=93 & 31(33.3 \%) & 36(38.7 \%) & 5(5.4 \%) & 21(22.6 \%) \\ \text { Total } & N=168 & 58(34.5 \%) & 77(45.8 \%) & 10(6 \%) & 23(13.7 \%)\end{array}$

Expression of Bmi1 and pAkt was evaluated by immunohistochemistry (IHC) on 168 human prostate cancers. Specimens were categorized in groups according to Gleason $\leq 7$ and Gleason $\geq 8$ and pathological stage $\mathrm{pT} 2$ and $\mathrm{pT} 3$.
Bmi1 is a phosphorylated protein $(20,21)$, yet the biological functions of its phosphorylation remain to be determined. To test whether Bmi1 and the PI3K/Akt pathway interact via phosphorylation of Bmi1, we first quantified the proportion of phosphorylated Bmi1 (pBmi1) relative to total Bmi1 in PTEN-deficient, pAkthigh cells (Jurkat cells as well as PCa cell lines PC3 and LNCaP) and PTENproficient, pAkt-low cells (MCF7 and U2OS cells as well as PCa cell line DU145). We observed that the pBmi1/ Bmi1 ratio was higher in PTEN-deficient, pAkt-high cells than in PTENproficient cells with lower pAkt levels (Figure 4A). This suggested that phosphorylation of Bmil might be directly

Bmi1 and pAkt coexpression was associated with advanced clinical stage (pT3, 22.6\%; pT2, 2.7\%; $P=0.002$; Figure $3 B$ and Table 1 ). In addition, when specimens were sorted according to both pathological grade and clinical stage, Bmi1 and pAkt were found to be preferentially coexpressed in the highest tumor grade category, with $30 \%$ incidence in Gleason $\geq 8$ and $\mathrm{pT} 3$ specimens (compared with $9.1 \%$ in Gleason $\leq 7$ and $\mathrm{pT} 3,5.3 \%$ in Gleason $\geq 8$ and $\mathrm{pT} 2$, and $1.8 \%$ in Gleason $\leq 7$ and pT2; $P=0.001$; Figure $3 \mathrm{C}$ and Table 1 ).

Given the heterogeneity and the focality of $\mathrm{PCa}$, it is common to observe the presence of tumor areas, PIN lesions, and normal prostate glands within the same histology slide. When this was the case, we scored PINs and normal glands for Bmi1 and pAkt expression. This revealed that Bmil was more frequently expressed in PINs than in normal glands. pAkt, which was rare but present in PINs, was virtually absent in nondiseased tissue, and always associated with Bmi1 (Supplemental Figure 5). This shows that expression of Bmi1 and pAkt and their association are unlikely to reflect variations between individuals, but rather represent a trait of increasing severity of neoplastic lesions. Together, these results indicate that upregulation of Bmi1 and activation of the PI3K/pAkt pathway are associated with adverse tumor characteristics, such as increasing pathological grade (poor cell differentiation) and worsening of clinical stage (extraprostatic extension).

Interestingly, we noticed that PIN lesions arising in PbCre4;Pten ${ }^{l o x P /+}$ prostates exhibited a slight elevation of Bmi1 expression compared with intact prostate ducts (Figure 1, $\mathrm{H}$ and J), which further highlights the parallels of the PbCre4;Bmi1 ${ }^{L S L}$; Pten $^{\text {loxP/+ }}$ mouse model with the human disease. We also observed that doxycycline-induced suppression of Bmi1 sensitized the human prostate adenocarcinoma cell line LNCaP (LNCaP-tetshBmil cell system) to growth inhibition induced by chemical inhibition of pAkt (Supplemental Figure 6), which suggests that Bmil and the PI3K pathway might be functionally linked in prostate cancer progression.

Akt phosphorylates Bmi1. Next, we investigated the molecular mechanisms underlying the interaction between Bmi1 and the PI3K pathway. Our initial observations in MEFs and in the PNT1a immortalized human prostate epithelial cell line revealed that modulation of Bmi1 did not significantly affect mRNA or protein levels of Pten, and vice versa (data not shown). This prompted us to test other possible mechanisms of interaction. influenced by PI3K/pAkt activity. To further test that hypothesis in prostate cells, we reconstituted PTEN in LNCaP cells. Expression of PTEN resulted in a dramatic decrease in pAkt levels, which was accompanied by a reduction in the levels of phosphorylated Bmi1 in favor of faster migrating, unphosphorylated Bmi1 species (Figure 4B). These data indicate that the PI3K/Akt pathway stimulates phosphorylation of Bmi1. Consistently, treatment of U2OS cells with PI3K inhibitors reduced the levels of phosphorylated Bmi1 (Supplemental Figure 7A).

Besides Akt, PI3K activates other proteins that may influence Bmi1 phosphorylation. Therefore, we sought to determine whether Akt itself is able to phosphorylate Bmi1. First, we treated PC3 and LNCaP cells with inhibitor VIII, which is specific for Akt. This resulted in a diminished $\mathrm{pBmi1/Bmi1}$ ratio from approximately $70 \%$ to approximately $55 \%$, down to the level observed in pAkt-low DU145 and PNT1a cells, whereas no effect of pAkt inhibition was observed in these later cells (Figure 4C). Similarly, siRNA-mediated knockdown of Akt 1 and Akt 2 isoforms in LNCaP cells also decreased the pBmi1/Bmi1 ratio (Supplemental Figure 7B). We conclude that Akt activity stimulates phosphorylation of Bmi1 in human PCa cells. Notably, in some instances, the reduced levels of pAkt were accompanied with an apparent increase in total amount of Bmi1, without affecting the global decrease of the $\mathrm{pBmi} 1 / \mathrm{Bmi} 1$ ratio. This is likely due to a selection effect of pAkt inhibition, in which cells with higher Bmi1 levels survive/proliferate more during Akt inhibitor treatment than do those with low Bmi1 levels (see Supplemental Figure 6). performed an in vitro Akt kinase assay in the presence of $[\gamma-32 \mathrm{P}]$ ATP. To ensure stability and proper folding of Bmi1 protein, and because the Bmi1 homolog Mel-18 was shown to be phosphorylated only when complexed with Ring1B (41), we used a recombinant Bmi1/Ring1B complex. As shown in Figure 5A, Akt specifically phosphorylated Bmi1 and its downstream direct substrate, GSK3. The negative control GST was not phosphorylated; neither was Ring1B, which migrates at a lower molecular weight. Bmi1 phosphorylation was not observed in the presence of an inactive Akt protein (data not shown).

In vivo, 9 independent phosphoproteomics studies, using different settings and biological systems, have identified 3 highly conserved phosphorylated serine residues (S251, S253, and S255)
Next, we addressed whether Bmi1 is a direct substrate for Akt. We 

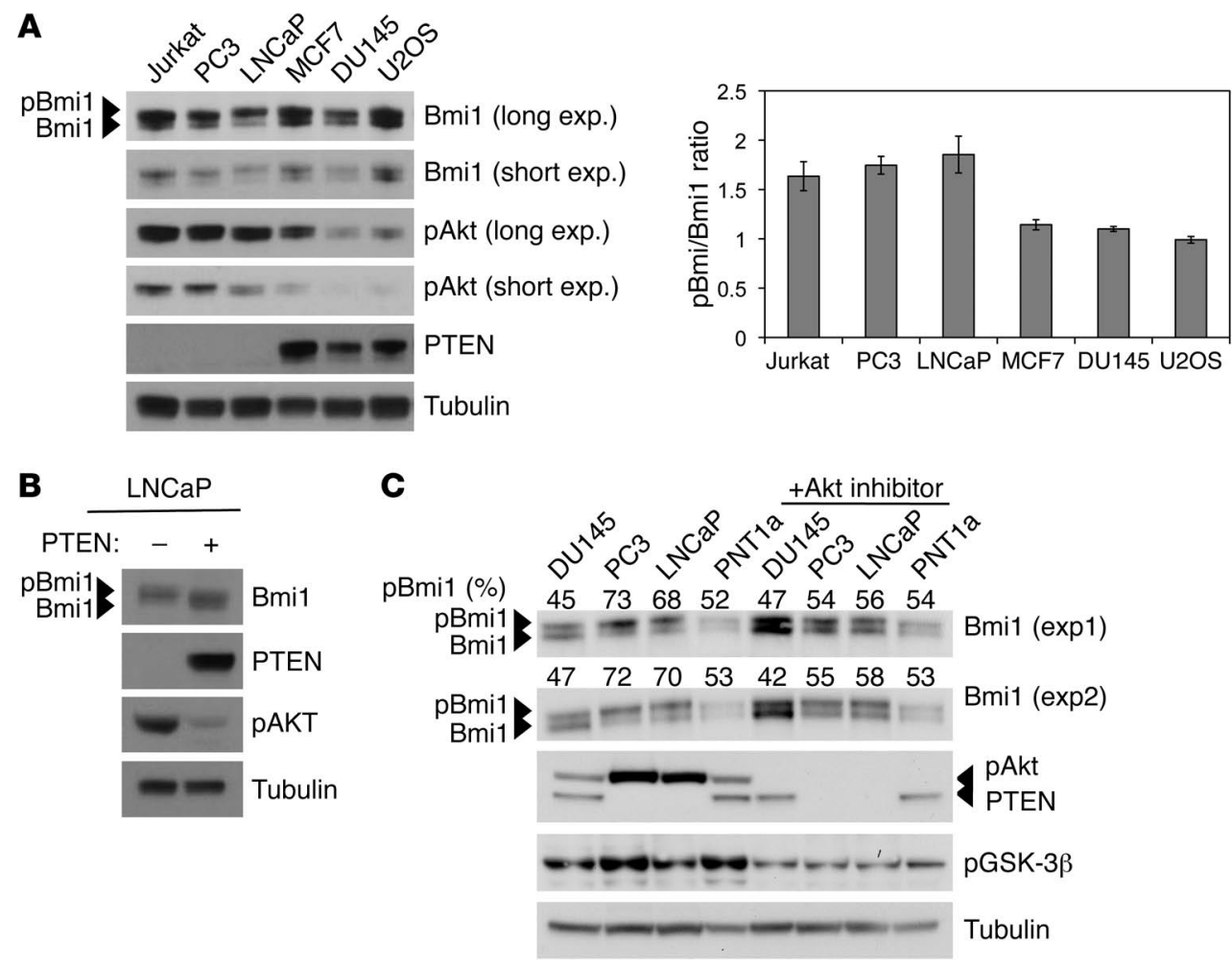

Figure 4

The Pten/PI3K/pAkt axis modulates Bmi1 phosphorylation. (A) Western blot analysis and relative quantification of pBmi1/Bmi1 ratio in Jurkat, PC3, and LNCaP Pten-deficient pAkt-high cells and in MCF7, DU145, and U2OS Pten-proficient pAkt-low cells. (B) Western blot showed that retrovirus-induced expression of PTEN in LNCaP cells inhibited pAkt and pBmi1. (C) Bmi1 phosphorylation was positively modulated by pAkt level. Bmi1 phosphorylation and pAkt and PTEN status in DU145, PC3, LNCaP, and PNT1a prostate cell lines either left untreated or treated with $5 \mu \mathrm{M}$ Akt inhibitor VIII (isozyme selective, Akt-1/2; Calbiochem) for 12 hours and refreshed 2 hours prior to lysis. pBmi1/Bmi1 ratios, quantified using ImageJ, are indicated within blots ( 2 separate experiments shown). The 3 lower panels corresponded to experiment 2 . Phosphorylation status of the Akt substrate GSK-3 $\beta$ (pSer9) is shown as a control for Akt activity. Tubulin was used as a loading control.

located in the C-terminal part of Bmi1 (refs. 22-30; the PhosphoSite Plus database, http://www.phosphosite.org; and Supplemental Figure 8, A and B). Thus, we produced recombinant Bmi1-3A mutant in which serines 251,253 , and 255 were replaced with alanine and subjected the mutant Bmi1-3A/Ring1B complex to a time course Akt kinase assay. Although the 3 serines do not lie within a characteristic Akt consensus site, their mutation resulted in a dramatic decrease in both kinetics and intensity of Akt-mediated phosphorylation of Bmi1 (Figure 5, A and B). This indicates that one or a combination of the 251,253, and 255 serines represent the main targets of Akt. However, as suggested by the remaining phosphorylation of the Bmi1-3A mutant, mutation of cognate serine targets may create secondary phosphorylation sites in in vitro conditions that were not identified in living cells. We thus resorted to use the Bmi1-3A mutant in our study. We then expressed HAtagged Bmi1-WT and Bmi1-3A proteins in LNCaP cells. Similar to the endogenous protein, HA-tagged Bmi1-WT migrated as 2 distinct species; in contrast, HA-tagged Bmi1-3A migrated as a single band (Figure 5C), which indicates that phosphorylation of this mutant is abolished in living cells. We conclude that Bmi1 is a bona fide phosphorylation target for activated Akt kinase both in vitro and in vivo and that the triad serine residues 251,253 , and 255 are critically involved in this process.

Phosphorylation of Bmi1 modulates $\mathrm{H} 2 \mathrm{~A}$ ubiquitination and DNA repair. In vitro, Bmi1 greatly stimulates Ring1B E3 ligase activity, and both proteins form an active core that is sufficient for the efficient ubiquitination of H2A-K119 $(15,16)$. To characterize how phosphorylation modulates Bmil function, we assayed the H2A ubiquitin ligase activity of the Bmi1/Ring1B complex pretreated with alkaline phosphatase (AP). Whereas Bmi1/Ring1B treated with inactivated AP efficiently ubiquitinated $\mathrm{H} 2 \mathrm{~A}$, this was greatly diminished by active AP treatment (Figure 6A). The Mel18/Ring1B complex (41) was used as a positive control. Interestingly, AP treatment did not affect the autopolyubiquitination of the complexes (Figure 6A).

Next, we assessed whether Akt-mediated phosphorylation of Bmi1 could stimulate Bmi1/ Ring1B ligase activity. As shown in Figure 6B, addition of active Akt kinase into the ubiquitination assay led to an increase of ubiquitinated $\mathrm{H} 2 \mathrm{~A}$ (Ub-H2A), whereas addition of inactive Akt had no effect. This indicates that phosphorylation of Bmi1 by Akt contributes to its stimulatory effect on Ring $1 \mathrm{~b}$ ubiquitin ligase activity in vitro. Since increased Ub-H2A levels influence global gene expression, we asked whether phosphorylation of Bmi1 affects global Ub-H2A levels in LNCaP cells. We used LNCaP-tetshBmil cells, which express a doxycycline-inducible shRNA specific to human Bmi1 (Supplemental Figure 6A), and reconstituted them with shRNA-insensitive mouse Bmi1-WT or Bmi1-3A retroviruses. In these cells, we observed that the global Ub-H2A level was not altered (Figure 6C). Similarly, chemical inhibition of Akt did not affect global Ub-H2A levels (Figure 6C). Consistently, we did not observe major changes in gene expression profiles of LNCaP-tetshBmi1 cells expressing Bmi1-WT or Bmi1-3A (data not shown). In addition, expression of Bmi1-3A mutant efficiently rescued $\mathrm{Bmi1}^{-/-}$ MEFs from premature senescence (31), and its capacity to repress the Ink4a/Arflocus remained intact (Figure 6D).

We therefore reasoned that, in vivo, Bmi1 phosphorylation might play a role independent of PRC1-mediated gene silencing. Recent studies have uncovered a function for Bmi1 in promoting the DNA damage response and reported its role for $\mathrm{H} 2 \mathrm{~A} / \gamma-\mathrm{H} 2 \mathrm{AX}$ ubiquitination at sites of DSBs (42-45). To determine whether 
A

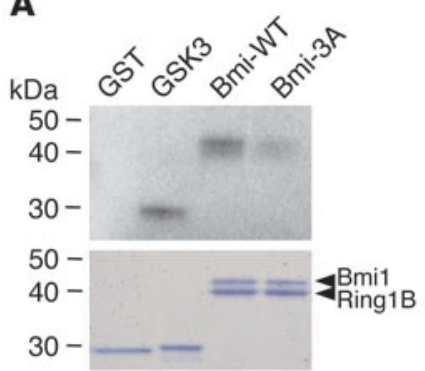

B

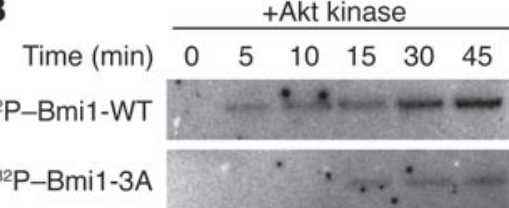

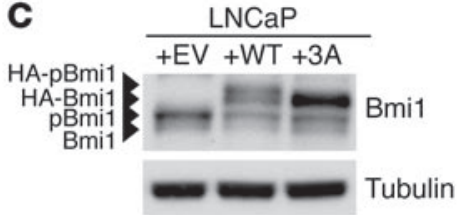

Figure 5

Bmi1 is phosphorylated by Akt. (A) In vitro Akt kinase assay with recombinant active Akt and Bmi1-WT/Ring1B or Bmi1-3A/Ring1B complex. Reactions were carried out for 40 minutes at $30^{\circ} \mathrm{C}$ in the presence of $[\gamma-32 P] A T P$. GST and GST-GSK3 $\alpha / \beta$ crosstide were used as negative and positive controls, respectively; phosphorylated proteins were visualized by autoradiography. A Coomassie-stained gel demonstrating equal loading is also shown. (B) Time course Akt kinase assay with recombinant active Akt and Bmi1-WT/Ring1B or Bmi1-3A/Ring1B complex. Equal amounts of substrate proteins and Akt were used in each assay. (C) Western blot analysis showing that HA-tagged Bmi1-3A was not phosphorylated in LNCaP cells.

phosphorylation of Bmi1 is required for DNA damage-induced H2A monoubiquitination, $\mathrm{Bmi1}^{-1-}$;Ink $4 a / A r f f^{-}$MEFs were reconstituted with vectors encoding either Bmi1-WT or Bmi-3A, and DNA DSBs were induced by UV laser scissor. As shown in Figure 7A, whereas both Bmi1-WT and Bmi1-3A were recruited to sites of DSBs, only Bmi1-WT was able to induce $\mathrm{H} 2 \mathrm{~A}$ ubiquitination. We also observed that in UV-irradiated cells, hyperphosphorylation of both Bmi1 and Akt was greatly induced (Figure 7B). Interestingly, Fraser et al. recently showed that Akt is phosphorylated in direct response to DNA DSBs, accumulates in the vicinity of IRinduced DSBs, colocalizes with DNA repair factors, and promotes DSBs repair (46). To determine whether UV-induced Bmi1-mediated $\mathrm{H} 2 \mathrm{~A}$ ubiquitination depends on Akt phosphosporylation, we repeated the UV laser scissor treatment in the presence of Akt inhibitor (Figure 7A). Strikingly, this experiment revealed that under Akt inhibition, while still recruited to the damaged sites, Bmi1 behaved like the Bmi1-3A phosphomutant and lost its E3 ligase activity toward $\mathrm{H} 2 \mathrm{~A}$.

To examine whether lack of Bmi1 phosphorylation affects HR repair of DSBs in cells, we used an I-Sce1 meganuclease homology-directed DNA repair assay that allows reporting of HR repair events by flow cytometry analysis of cellular GFP (47). Reconstitution of Bmi1-WT expression in Bmil-depleted
A

AP AP inactivated
Nucleosome Ubiquitin

C LNCaP-tet-shBmi1 +Dox

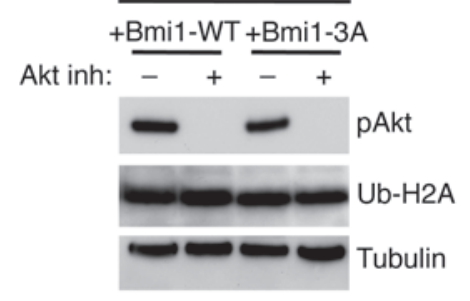

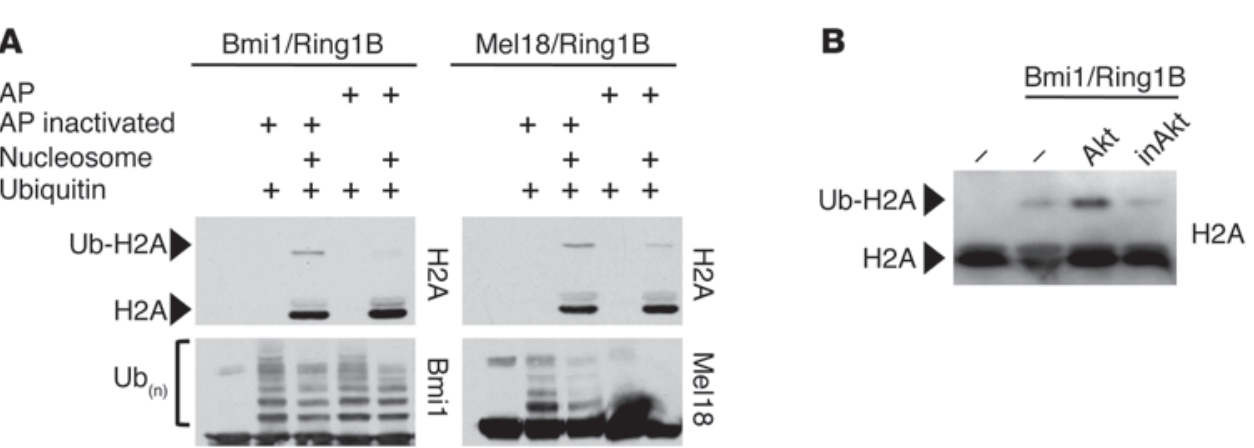

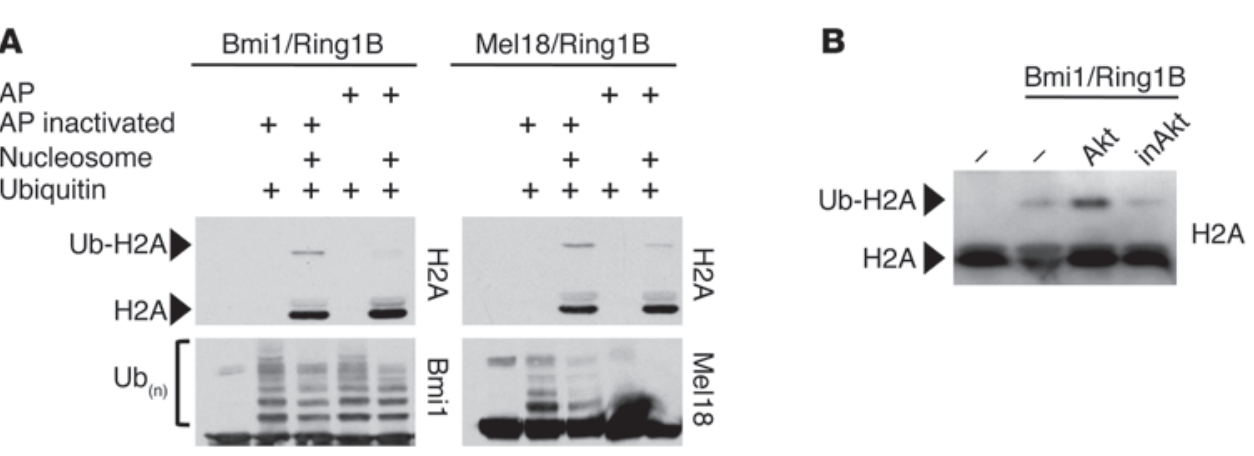

U2OS cells led to $3.3 \%$ of cells being $\mathrm{GFP}^{+}$, a 2.4-fold induction over empty vector- and Bmi1-3A-transfected cells (Figure 8A), which indicates that impairment of Bmi1 phosphorylation leads to defects in DSB-promoted HR repair. 
A

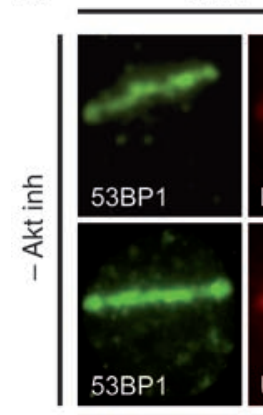

Bmi1 ${ }^{-1} ;$ Ink4a/Arf $/-$ MEFs +Bmi1-WT
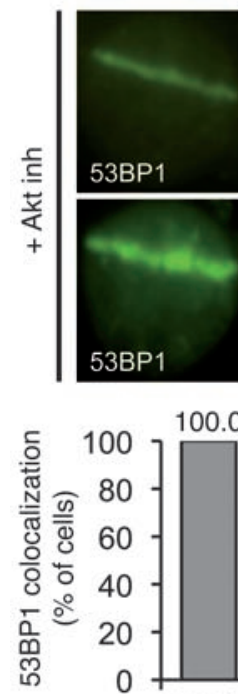

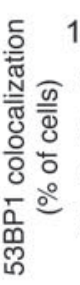
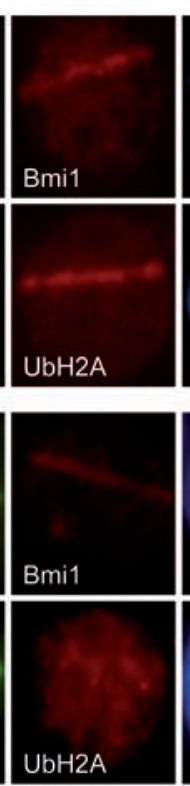
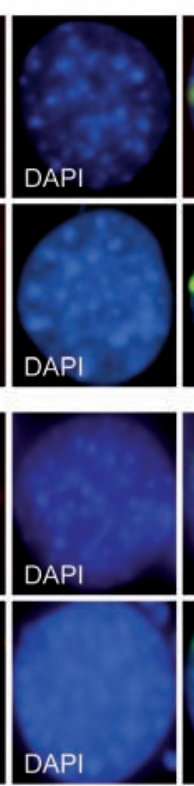

100.0
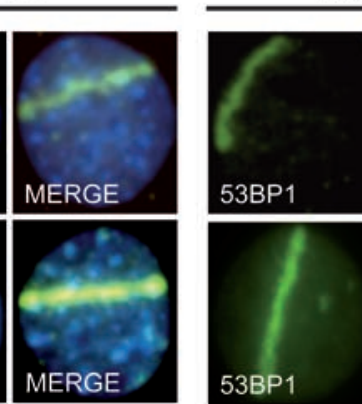

$\mathrm{Bmi1}^{-+} ;$Ink4a/Arf ${ }^{-}$MEFs $+B m i 1-3 \mathrm{~A}$
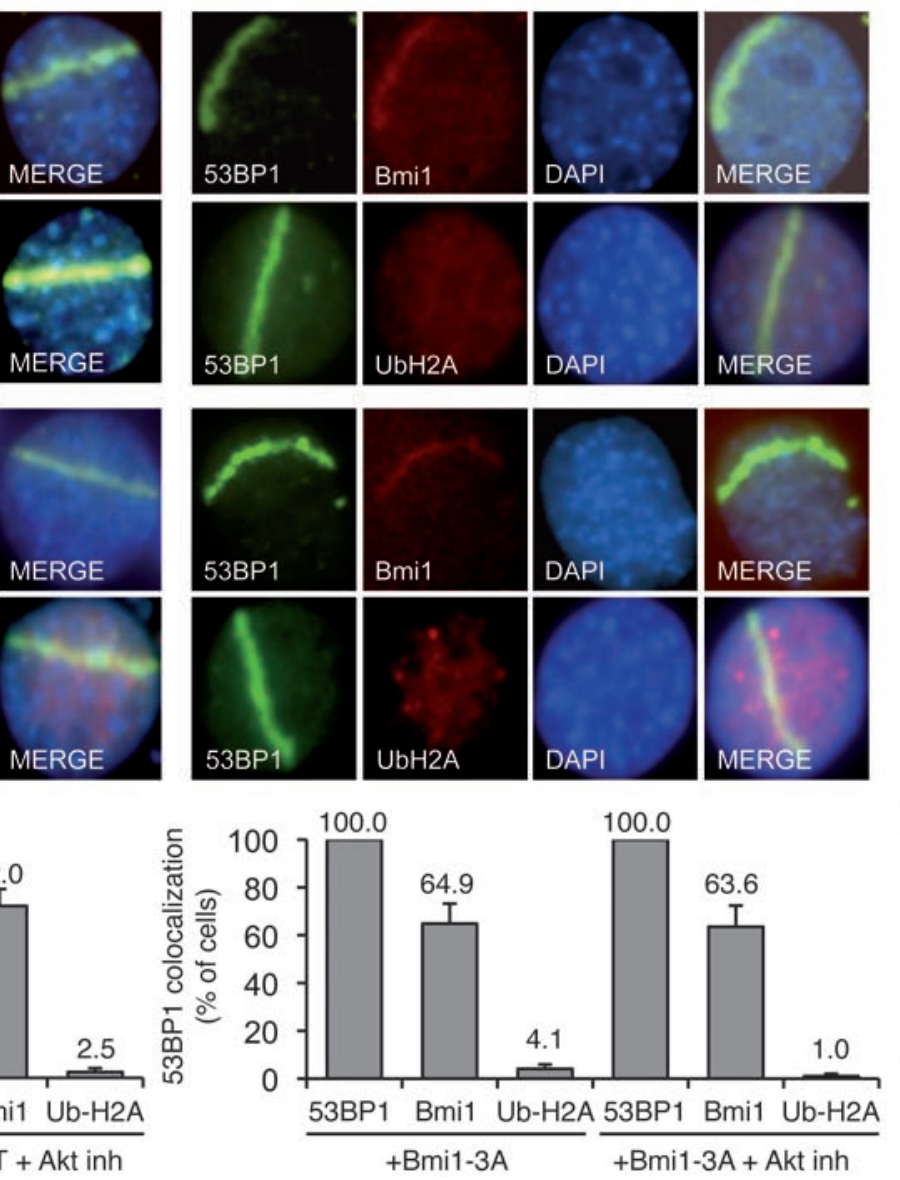

B

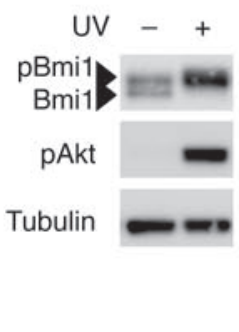

Figure 7

Phosphorylation of Bmi1 stimulates H2A ubiquitination at DNA DSBs. (A) Phosphorylation of Bmi1 was required for $\mathrm{H} 2 \mathrm{~A}$ ubiquitination at laser scissors-induced DNA breaks. Bmi1---;Ink4a/Arf-- MEFs were reconstituted with either Bmi1-WT or Bmi1-3A, then treated with UV laser scissors in the presence or absence of Akt inhibitor. Cells were then processed for immunofluorescence using antibodies to 53BP1 and either Bmi1 or Ub$\mathrm{H} 2 \mathrm{~A}$. Percent cells with laser scissors-induced 53BP1 localization is also shown, demonstrating colocalization of Bmi1 and Ub-H2A with 53BP1 in each of these conditions. (B) Western blot showed that global UV irradiation $\left(20 \mathrm{~J} / \mathrm{m}^{2}\right)$ induced phosphorylation of both Bmi1 and Akt.

To extend our results, we examined HR in our LNCaP-tetshBmil cell system by measuring the frequency of spontaneous sister chromatid exchanges (SCEs), a commonly used index of chromosomal stability. In doxycycline-treated LNCaP-tetshBmi1 cells, expression of Bmi1-WT, but not Bmi1-3A, led to a statistically significant, 2.3-fold increase of spontaneous SCEs (Figure 8B). Importantly, this effect was dependent on Akt activity, since Akt inhibition completely abolished the effect of Bmi1WT, reducing the number of SCEs to basal levels (Figure 8B). These results prompted us to assess the level of DNA damage in our mouse and human prostate tumor sets using $\gamma-\mathrm{H} 2 \mathrm{AX}$ immunohistochemistry. Tumor areas of PbCre4;Bmi1 ${ }^{\text {LSL }}$;Pten ${ }^{\text {lox }} /++$ mice and Bmi1/pAkt positive human samples exhibited high levels of DNA damage, which was absent - or present to a much lesser extent - in control mouse prostates and Bmi1/pAkt single-positive and double-negative human samples (Figure 8C). Together, these data showed that Akt-dependent phosphorylation of Bmi1 was required for DNA damage-induced H2A ubiquitination and efficient DNA repair and that sustained phosphorylation of Bmi1 by Akt led to genomic instability, as assessed by high HR rates.

Phosphorylation of Bmi1 increases its oncogenic potential in vitro and in vivo. To determine whether Bmi1 phosphorylation influences its oncogenic function, we assessed the transformation potential of Bmi1-3A in a soft agar colony formation assay using Ink4a/ Arf ${ }^{\prime-}$ MEFs overexpressing c-Myc. As expected, Bmi1-WT readily conferred anchorage-independent cell growth, whereas Bmi1-3A was severely impaired in this process (Figure 9A), which indicates that Bmi1 phosphorylation is a requisite for its transformation ability.

To address the relevance of these findings in the context of PCa, we assessed the ability of Bmi1-WT or Bmi1-3A to rescue the growth defect observed in doxycycline-treated LNCaP-tetshBmi1 cells. LNCaP-tet-shBmi1 cells were then superinfected with retroviruses encoding shRNA-insensitive mouse Bmi1-WT or Bmi1-3A. Ectopically expressed Bmi1-WT significantly rescued the growth defect of LNCaP-tet-shBmi1, whereas expression of Bmi1-3A remained ineffective (Figure 9B). This effect 
A
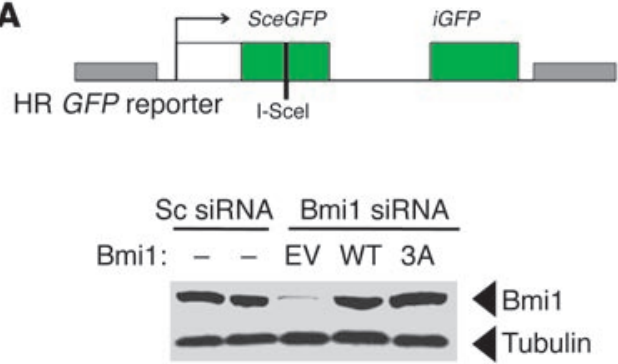

B

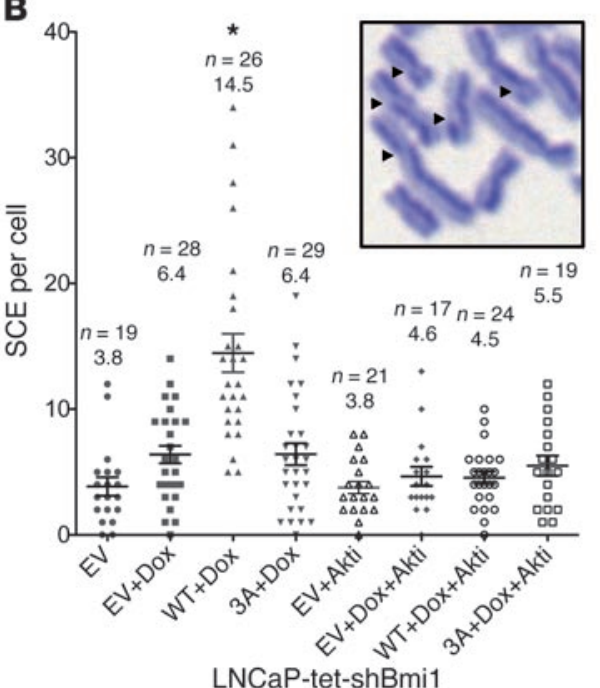

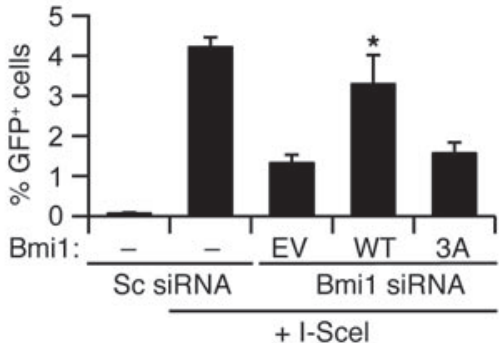

C Mouse prostates/ $\gamma \mathrm{H} 2 \mathrm{AX}$

PbCre4 PbCre4;Bmi1 $\quad$ LSL PbCre4;Pten ${ }^{\text {loxP/+ }}$ PbCre4;Bmi1 ${ }^{\text {LSL }}$;

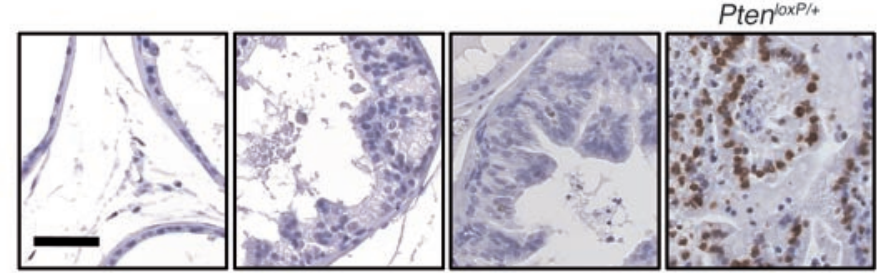

Human prostates $/ \gamma \mathrm{H} 2 \mathrm{AX}$

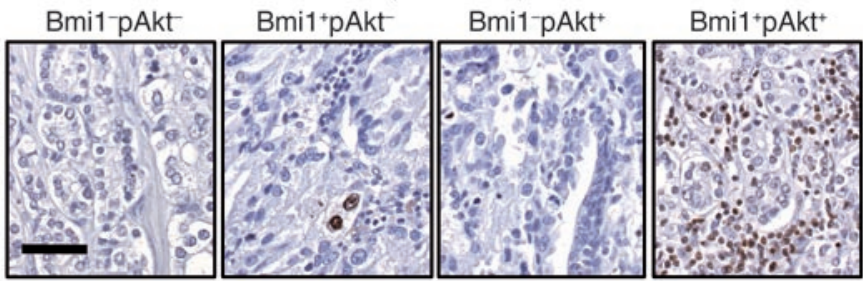

\section{Figure 8}

Phosphorylation of Bmi1 enhances HR and genomic instability. (A) Phosphorylation of Bmi1 was required for efficient DNA DSB repair by HR. The HR GFP reporter is stably integrated into the genome of U2OS cell line. SceGFP is a GFP gene that contains an I-Scel endonuclease site within the coding region. Cleavage of the I-Scel site in vivo and repair by HR directed by the downstream iGFP repeat results in GFP+ cells. HR GFP U2OS cells were transfected with scrambled (Sc) siRNA or Bmi1 siRNA reconstituted with constructs expressing mouse empty vector, Bmi1-WT, or Bmi1-3A. Where indicated, cells were also transfected with an I-Scel expression vector. After 24 hours, the percentage of GFP + cells was measured by flow cytometry. Data are means \pm SD of 3 independent experiments. ${ }^{*} P=0.006$ versus Bmi 1 -WT, unpaired $t$ test. (B) Frequency of spontaneous SCEs in doxycycline-treated LNCaP-tet-shBmi1 prostate cancer cells infected with retroviruses expressing Bmi1-WT, Bmi1-3A, or control empty vector. SCEs were increased 2.3-fold with Bmi1-WT, but not Bmi1-3A, overexpression $\left({ }^{*} P<0.0001\right.$, unpaired $t$ test). Akt inhibition (Akti) suppressed Bmi1-WT-induced SCEs. Data are means \pm SEM. Examples of SCE events (arrowheads) are shown in the inset, which depicts subsets of chromosomes in a metaphase spread labeled for SCE analysis (original magnification, $\times 200)$. (C) Immunohistochemistry for $\gamma \mathrm{H} 2 \mathrm{AX}$ revealed greater DNA damage in tumor areas of PbCre4;Bmi1 ${ }_{\text {LSL; } P \text { ten }}^{\text {loxP/+ }}$ mutant prostates $(n=27)$ and Bmi1+pAkt+ human samples $(n=77)$. Scale bars: $100 \mu \mathrm{m}$.

was partly dependent on the expression of the cell cycle regulator p21 CDKN1A (Supplemental Figure 9A). Consistently, when injected subcutaneously in Balb/c nude mice, LNCaPs expressing Bmi1WT generated significantly larger tumors than parental and Bmi1-3A-expressing LNCaPs (Figure 9C and Supplemental Figure 9B). In addition, we performed orthotopic transplant experiments using immortalized human prostate epithelial cells (hPrECs). hPrECs expressing SV40 LT, hTERT, c-Myc, and PI3K (LHMK) were previously shown to have limited tumor-forming capability (48). In our study, we used hPrECs that express SV40 LT, hTERT, c-Myc, and shPTEN (LHMshP) (Supplemental Figure 9C). LHMshP hPrECs also displayed limited tumor-forming ability (Figure 9D). However, overexpression of Bmi1-WT in these cells increased their tumorigenicity 2.5 -fold relative to empty vector-expressing LHMshP hPrECs (Figure 9D). Expression of Bmi1-3A in LHMshP hPrECs only had a moderate effect on tumorigenicity, which indicates that Bmi1 phosphorylation is required for efficient stimulation of PCa cell growth.

Finally, we investigated whether these findings could be extended to other tumor types. It is well established that Bmi1 cooperates with c-Myc to induce lymphoma in mice $(18,33)$. We therefore tested the ability of Bmi1-3A to accelerate lymphomagenesis in a transduction/reconstitution assay using the E $\mu$-Myc lymphoma model (49). E $\mu$-Myc-derived hematopoietic stem and progenitor cells (HSPCs) transduced with a retrovirus encoding Bmi1-WT or Bmi1-3A were engrafted into lethally irradiated syngeneic mice. Animals reconstituted with E $\mu$-Myc control cells developed lymphoma with a median survival of 88 days (Figure 9E and Supplemental Figure 9D). As expected, overexpression of Bmi1-WT resulted in significant acceleration of lymphomagenesis compared with control (median survival, 61 days; $P=0.0064$ ). In contrast, overexpression of Bmi1-3A had no significant effect on lymphoma 
A

B
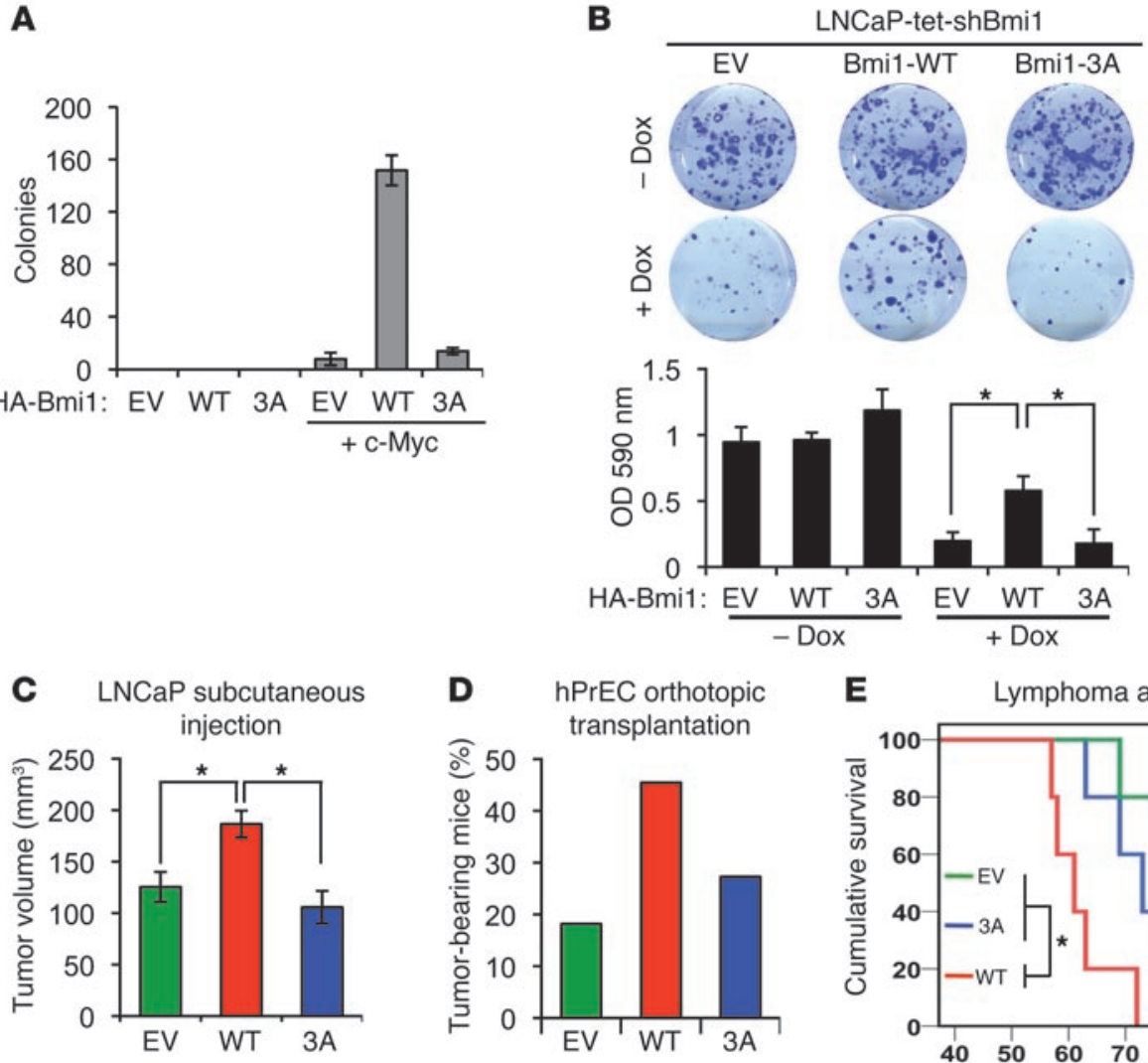

E

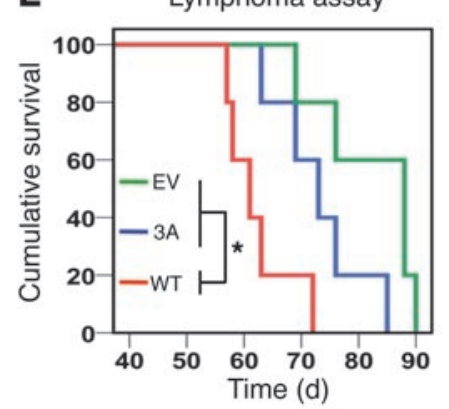

Figure 9

Phosphorylation of Bmi1 regulates its oncogenic potential. (A) Ink4a/Arf ${ }^{-1}$ MEFs were infected with the indicated retroviral constructs and assayed for anchorage-independent growth (soft agar) for 4 weeks. Data (mean $\pm \mathrm{SD}$ ) represent number of colonies from 2 independent experiments performed in triplicate. (B) $\mathrm{Bmi} 1$ reconstitution in human prostate cancer cells. LNCaP-tet-shBmi1 cells infected with the indicated retroviral constructs were incubated for 8 days in the presence of doxycycline and then stained with crystal violet; cell growth was estimated by OD quantification of the crystal violet at $590 \mathrm{~nm}$. Data are mean \pm SD of 3 independent experiments. (C) LNCaP-tet-shBmi1 cells infected with the indicated retroviral constructs were injected subcutaneously into nude mice. Tumor size was measured after 4 weeks. Data are means $\pm \mathrm{SE}$ $(n \geq 12) . P=0.008$, empty vector versus Bmi1-WT; $P=0.002$, Bmi1-3A versus Bmi1-WT. (D) Percent tumor-bearing mice 3 months after orthotopic transplantation with LHMshP hPrECs ( $n=11$ per group). (E) Kaplan-Meier analysis of overall lymphoma-free survival. E $\mu$-Myc fetal liver hematopoietic cells were infected with the indicated retroviral constructs and injected intravenously to reconstitute lethally irradiated recipient mice ( $n=5$ per group). $P=0.062$, empty vector versus Bmi1-3A; $P=0.006$, empty vector versus Bmi1-WT; $P=0.026$, Bmi1-3A versus Bmi1-WT. ${ }^{*} P<0.05$.

onset compared with control (median survival, 73 days; $P=0.061$; Figure 9E), which indicates that phosphorylation of Bmi1 constitutes an important signal for its in vivo lymphomagenic potential as well. Together, these results show that Bmil phosphorylation, by Akt and possibly other kinases, plays a major role in the modulation of Bmi1 oncogenic potential in vitro and in vivo.

\section{Discussion}

Bmil overexpression cooperates with PTEN haploinsufficiency in invasive prostate cancer. Although previous studies suggested a positive correlation between Bmi1 expression and PCa progression $(11,13)$, a causal role was not demonstrated experimentally. By targeting Bmil overexpression to the murine prostate, we showed that it was sufficient to initiate the first steps of prostate carcinogenesis. Hence, this established a direct role for the PcG epigenetic silencer Bmi1 in the etiology of prostate cancer.

We found that Bmil and PI3K/Akt were coactivated in high-grade human PCa. Consistently, PbCre4;Bmi1 ${ }^{\text {LSL }}$;Pten ${ }^{\text {loxP/+ }}$ animals developed invasive prostate adenocarcinoma that recapitulated features of human disease: medium/late onset, exhibition of neoplastic precursor stages, and multifocality. Moreover, the cancerous lesions developed for the most part in the dorsolateral lobe of the mouse prostate, which is considered homologous to the human peripheral zone from which most cancers arise (50). In PbCre4;Bmi1 LSL;Pten ${ }^{\text {loxP/+ }}$ mice, progression to metastatic disease was not observed, suggestive of the requirement of additional genetic and/or epigenetic events. In this regard, another PcG member, $E Z H 2$, which is expressed in high-grade and metastatic $\mathrm{PCa}$ (10), promotes metastasis in an orthotopic transplantation PCa model (51). It will be of interest to address whether prostate-targeted EZH2 expression can induce metastatic conversion of PbCre4;Bmi1 ${ }^{\text {LSL; }}$; Pten $^{\text {loxP/+}}$ tumors.

Both Bmi1 and PI3K/Akt signaling pathways are activated in PCa stem-like cell populations (52). Recently, Lukacs and colleagues reported that Bmil inhibition attenuates the growth of Pten-null prostate cancer cells and that Bmi1 expression is required for the maintenance of normal stem-like cells (53). Our studies support and extend these findings by describing underlying molecular mechanisms and prompt new investigations using PbCre4;Bmil ${ }^{L S L}$ and PbCre4;Bmi1 ${ }^{\text {LSL; }}$ Pten ${ }^{\text {loxP/+ }}$ models to further characterize the cancer cell of origin and pathways involved in normal and cancer stem cell maintenance in the prostate epithelium.

Regulation of Bmi1 oncogenic potential by phosphorylation. Besides their increased levels in a variety of cancers, including in the prostate, PcG proteins also undergo posttranslational modifications (54), the consequences of which remain largely unknown. Recent studies have uncovered a function for Bmi1 in DNA damage response and demonstrated its role in $\mathrm{H} 2 \mathrm{~A} / \gamma-\mathrm{H} 2 \mathrm{AX}$ ubiquitination at DSBs and for efficient DNA damage repair (42-45, 55). Here, we revealed an important role for Bmil phosphorylation in mediating $\mathrm{HR}$ repair through the stimulation of $\mathrm{H} 2 \mathrm{~A}$ ubiquitina- 


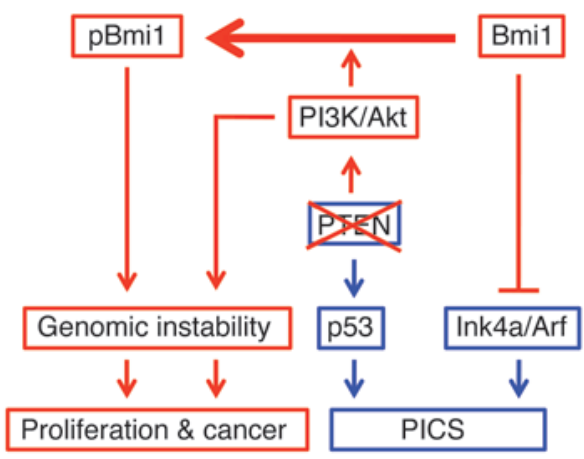

\section{Figure 10}

Model for Bmi1 and PTEN/PI3K/Akt collaboration in prostate cancer. Schematic model illustrates the synergy of Bmi1 and PTEN/PI3K/Akt pathways in prostate tumorigenesis. PTEN loss activates the PI3K/ Akt pathway and positively modulates p53 levels, leading to the activation of PTEN-induced cellular senescence (PICS). Bmi1 counters cellular senescence by repressing Ink4a/Arf. Upon Akt-mediated phosphorylation, Bmi1 acts mainly through an Ink4a/Arf-independent mechanism to promote prostate carcinogenesis in collaboration with the PI3K/Akt pathway.

tion at damaged sites. Consistent with Akt activation in response to DNA damage and its accumulation at DSBs (46), we demonstrated that Akt plays an important role in the local stimulation of Bmi1 ubiquitin ligase activity on $\mathrm{H} 2 \mathrm{~A}$. Although our in vitro experiments showed direct phosphorylation of Bmil by Akt, we cannot exclude that Akt-mediated phosphorylation of Bmi1 in vivo may be indirect. Nevertheless, our findings suggest a great degree of complexity in the regulation of Bmi1 and validate the existence of cross-talk between Bmi1/PI3K/Akt and DNA damage repair pathways. It is critical to understand the mechanisms underlying this cross-talk for the design of rational therapeutic strategies that could disrupt these connections for the radiosensitization of tumors with hyperactivated Bmi1 and PI3K/Akt signaling. From its earliest stages, cancer development of various tumor types is associated with DNA replication stress, which leads to DNA strand breaks. Both deficient and exacerbated HR activity determine genome instability $(56,57)$, which is directly implicated in tumor progression (58). Although DNA damage repair per se would not cause cancer, it is likely that over time, constitutively increased DNA damage repair activity in combination with prosurvival signals can enable cancerous and/or precancerous cells to survive disastrous DNA damage and, in an insidious loop, allow accumulation of mutation events, increasing overall genomic instability and cancer progression.

Elevated DNA repair capacity in cancer cells is also known to limit the efficacy of radiation therapy. In line with this, Bmi1 confers radioresistance to normal and cancerous cells $(43,44)$. The PI3K/Akt pathway is also involved in increased radioresistance (59-62). This raises the possibility that patients with advanced prostate cancer showing elevated Bmi1 and pAkt may benefit from pharmacological inhibition of Bmi1 and/or Akt combined with radiation therapy.

Loss of PTEN leads to genomic instability, and physiological activation of Akt (as a result of genetic inactivation of PTEN) was previously shown to suppress DNA damage processing and checkpoint activation in late $\mathrm{G}_{2}(63)$. Ginjala et al. reported that loss of Bmi1 leads to accumulation of cells in $\mathrm{G}_{2} / \mathrm{M}$ associated with DNA damage checkpoint activation (45). Therefore, increase of Bmi1 level and its phosphorylation, together with Akt activation, may synergize to increase their potential to bypass $\mathrm{G}_{2} / \mathrm{M}$ checkpoint arrest in the face of genotoxic damage and increase genomic instability, which is a hallmark of neoplastic progression.

Phosphorylation of Bmi1 is likely to be involved in the finetuning of its activity in a gene- or context-specific manner, rather than in the global regulation of PRC1 function. Indeed, mutation of Bmi1 phosphorylated sites or Akt inhibition did not affect the global levels of Ub-H2A or the ability to repress the Ink4a/Arflocus, and we did not observe major differences in global gene expression profiles of Bmi1-3A- versus Bmi1-WT-expressing LNCaPs, although some changes in potentially important cell cycle regulators were observed (such as p21; Supplemental Figure 8A). This phosphoregulation may serve to separate Bmi1-repressive and repair functions and to rapidly modulate its enzymatic activity in a local, specific context, while leaving global H2A ubiquitination and PRC1-mediated gene repression intact. Such a "local" mode of regulation benefits from its reversibility, as dephosphorylation of Bmi1 may occur readily after repair along with $\mathrm{H} 2 \mathrm{~A}$ deubiquitination at repaired DSBs, while global Ub-H2A levels must remain stable.

Acute loss of Pten in MEFs and in the prostate triggers oncogene-induced senescence via upregulation of the Ink $4 a / A r f$ locus and the p53 pathway (64). Overexpression of Bmi1 in MEFs bypasses Pten loss-induced senescence and is accompanied by Ink4a/Arf downregulation (Supplemental Figure 10), which suggests that part of Bmi1's oncogenic activity involves its repression function, notably toward Ink4a/Arf. However, the involvement of Ink $4 a / A r f$ in prostate carcinogenesis is still debated, since Arf deficiency partially inhibits the prostate cancer phenotype of Pten-deficient mice (65) and prostates lacking Ink4a/Arf do not develop cancerous/precancerous lesions over a long period of time (66), in line with a notable rarity of INK4a/ $A R F$ inactivation in human PCa. It was suggested that Bmil may stimulate human PCa cell growth in part via repression of the INK4a/ARF locus (67). As far as we have observed, the expression of that locus was low to absent in most PCa cells tested, and Bmi1 expression (either WT or $3 \mathrm{~A}$ ) did not further repress it (data not shown).

Our study revealed that Bmi1 induced PIN lesions, which suggests that the role of Bmi1 in PCa is more complex and goes beyond Ink4a/Arf repression and is, at least in part, uncoupled from its antisenescence activity and modulated by its phosphorylation (Figure 10). We propose that the oncogenic potential of Bmi1 is regulated at both transcriptional and posttranslational levels. Repression of senescence by Bmi1 is regulated by its expression level, while effects on cellular transformation also rely in part on other mechanisms, such as Bmi1 phosphorylation and possibly its consequences on DNA damage processing. Consistently, heterozygosity for $\mathrm{Bmi1}$ reduces lymphomagenesis in E $\mu$-Myc mice in an Ink4a/Arf-dependent manner (33), and Bmil deficiency impedes tumor growth in an Ink4a/Arf-independent manner in a mouse model for glioma (34). Therefore, more emphasis should be put on the role of Bmi1 posttranslational modifications and how they modulate its oncogenic functions. Since concomitant elevation of Bmi1 and activation of the PI3K/Akt pathway are traits of patients with advanced $\mathrm{PCa}$, assessing Bmi1 phosphorylation status may be important to predict disease outcome. 


\section{Methods}

Mice. Bmi ${ }^{L S L}$ mice (see Supplemental Methods) were crossed with PbCre4 (36) and $P \operatorname{ten}^{l o x P}(68)$ FVB mice to generate the cohort used in the present study.

For the LNCaP xenograft assay, 7 -week-old male Balb/c nude mice were subcutaneously injected with $2 \times 10^{6}$ cells in PBS with 50\% Matrigel (BD) in a volume of $500 \mu$ l. Tumor growth was monitored daily, and mice were sacrificed after 4 weeks. Tumor size was determined by direct measurement after dissection, and samples were fixed in formalin for histopathological analysis.

Parental hPrEC cells were a gift from W.C. Hahn (Dana-Farber Cancer Institute, Boston, Massachusetts, USA). For orthotopic transplantation of LHMshP hPrECs, $10^{6}$ cells $(10 \mu \mathrm{l})$ were mixed with Matrigel $(10 \mu \mathrm{l})$ and injected into the anterior prostate lobe of 8 -week-old nude mature males. Injected cells expressed luciferase, and tumor outgrowths were monitored weekly by IVIS bioluminescence Imaging System.

The lymphoma assay was performed essentially as described previously (69). Hematopoietic E $\mu$-Myc fetal liver cells were infected with retroviruses encoding Bmi1-WT or Bmi1-3A and intravenously injected into lethally irradiated mice. Reconstituted animals (5 per group) were monitored for illness by lymph node palpation and overall morbidity. Overall survival was defined as the time from stem cell reconstitution until the animal reached morbidity, and statistical analysis was performed using the Mantel-Cox test.

Histopathology and immunohistochemistry. Prostate lobes were individually evaluated for pathology according to the guidelines of the Bar Harbor Mouse Models of Human Cancer Consortium Prostate Pathology committee. IHC was done according to the manufacturers' recommendations. The following primary antibodies were used: Bmi1 mAb (clone F6; Upstate); pAkt (Ser473) (D9E; Cell Signaling); Cytokeratin14 (PRB155P; Babco/Covance); p63 (4A4; Santa Cruz); synaptophysin (A0010; Dako); cytokeratin 8 (Troma-1; Developmental Study Hybridoma Bank, University of Iowa); androgen receptor (sc-816; Santa Cruz); E-cadherin (36/EC; BD Transduction Laboratories); Ki-67 (TEC-3; Dako). Secondary antibodies were coupled to Strep-AB complex, and immunodetection was performed using Liquid DAB Substrate Chromogen System (Dako) and counterstained with hematoxylin.

Human tissue specimens. 168 formalin-fixed paraffin-embedded primary PCa specimens were obtained from the Nederlands Kanker InstituutAntoni van Leeuwenhoek (NKI-AVL). Pathohistological characterization and grading of the 168 PCa cases was assessed by the Division of Human Pathology, NKI-AVL, as part of routine clinical management. The tissue cohort was divided by both Gleason score ( $\leq 7$ and $\geq 8$ ) and TNM tumor staging (pT2 and pT3). Scoring of immunostained slides was done accord- ing to the percentage of tumor cells exhibiting nuclear (Bmi1) or nuclear/ cytoplasmic (S473-pAkt) staining. To define positive and negative expressors, we used a cutoff of $30 \%$ or more positive tumor cells. Tumors were scored as double positive if they fulfilled above criterions in the same glandular area of 2 consecutive sections.

Statistics. Data are expressed as mean \pm SD. Statistical significance was determined by 2-tailed Student's $t$ test, except for human tissue specimen analysis, for which $\chi^{2}$ test was used. A $P$ value less than 0.05 was considered statistically significant.

Study approval. All experiments involving the use of animals, including for the generation of MEFs, were reviewed and approved by the local Animal Experiment Committee (DEC NKI, Amsterdam) and were conducted in compliance with the animal welfare regulations of The Netherlands. Paraffin-embedded human PCa tissue samples were provided by the tissue bank of the NKI in accordance with the regulations of the tissue bank and with the approval of the ethics committee of the NKI. Patients provided informed consent for the use of tissue.

\section{Acknowledgments}

We thank A. Berns, E. Citterio, A. Sparmann, and G. Gargiulo for help, critical reading, and editing of the manuscript and the Human and Animal Pathology Departments for tissue management. We are indebted to our colleagues of the NKI animal facility for animal husbandry. K. Nacerddine was supported by an EMBO long-term fellowship and a Dutch Cancer Society (KWF Kankerbestrijding) grant. M. van Lohuizen was supported by a CBG grant. J.B. Beaudry was supported by ESTOOLS FP7 EU grant and by the CBG.

Received for publication February 7, 2011, and accepted in revised form February 29, 2012.

Address correspondence to: Maarten van Lohuizen, Division of Molecular Genetics and Center for Biomedical Genetics, Netherlands Cancer Institute, Plesmanlaan 121, 1066 CX Amsterdam, The Netherlands. Phone: 31.20.512.2030; Fax: 31.20.512.2011; E-mail:m.v.lohuizen@nki.nl.

Jean-Bernard Beaudry's present address is: de Duve Institute and Université catholique de Louvain, Brussels, Belgium.

Bart Westerman's present address is: Department of Human Genetics, Academic Medical Center, Amsterdam, The Netherlands.
1. Jemal A, Siegel R, Ward E, Hao Y, Xu J, Thun MJ. Cancer statistics, 2009. CA Cancer J Clin. 2009; 59(4):225-249.

2. Dong JT. Prevalent mutations in prostate cancer. J Cell Biochem. 2006;97(3):433-447.

3. Whang YE, et al. Inactivation of the tumor suppressor PTEN/MMAC1 in advanced human prostate cancer through loss of expression. Proc Natl Acad Sci U S A. 1998;95(9):5246-5250.

4. Gray IC, et al. Mutation and expression analysis of the putative prostate tumour-suppressor gene PTEN. BrJ Cancer. 1998;78(10):1296-1300.

5. Dahia PL. PTEN, a unique tumor suppressor gene. Endocr Relat Cancer. 2000;7(2):115-129.

6. Wu X, Senechal K, Neshat MS, Whang YE, Sawyers CL. The PTEN/MMAC1 tumor suppressor phosphatase functions as a negative regulator of the phosphoinositide 3-kinase/Akt pathway. Proc Natl Acad Sci U S A. 1998;95(26):15587-15591.

7. Cooney KA, et al. Absence of PTEN germ-line mutations in men with a potential inherited predisposition to prostate cancer. Clin Cancer Res. 1999;
5(6):1387-1391.

8. Forrest MS, et al. No evidence of germline PTEN mutations in familial prostate cancer. J Med Genet. 2000;37(3):210-212.

9. Schulz WA, Hatina J. Epigenetics of prostate cancer: beyond DNA methylation. J Cell Mol Med. 2006; 10(1):100-125.

10. Varambally $\mathrm{S}$, et al. The polycomb group protein $\mathrm{EZH} 2$ is involved in progression of prostate cancer. Nature. 2002;419(6907):624-629.

11. Glinsky GV, Berezovska O, Glinskii AB. Microarray analysis identifies a death-from-cancer signature predicting therapy failure in patients with multiple types of cancer.J Clin Invest. 2005;115(6):1503-1521.

12. Berezovska OP, Glinskii AB, Yang Z, Li X-M, Hoffman RM, Glinsky GV. Essential role for activation of the Polycomb group (PcG) protein chromatin silencing pathway in metastatic prostate cancer. Cell Cycle. 2006;5(16):1886-1901.

13. van Leenders $G$, et al. Polycomb-group oncogenes EZH2, BMI1, and RING1 are overexpressed in prostate cancer with adverse pathologic and clini- cal features. Eur Urol. 2007;52(2):455-463.

14. $\mathrm{Yu}$ J, et al. A polycomb repression signature in metastatic prostate cancer predicts cancer outcome. Cancer Res. 2007;67(22):10657-10663.

15. Cao R, Tsukada Y-I, Zhang Y. Role of Bmi-1 and Ring1Ain H2Aubiquitylation and Hox gene silencing. Mol Cell. 2005;20(6):845-854.

16. Buchwald G, van der Stoop P, Weichenrieder O, Perrakis A, van Lohuizen M, Sixma TK. Structure and E3-ligase activity of the Ring-Ring complex of polycomb proteins Bmi1 and Ring $1 \mathrm{~b}$. EMBO J. 2006;25(11):2465-2474.

17. Wang $\mathrm{H}$, et al. Role of histone $\mathrm{H} 2 \mathrm{~A}$ ubiquitination in Polycomb silencing. Nature. 2004; 431(7010):873-878.

18. van Lohuizen M, Verbeek S, Scheijen B, Wientjens E, van der Gulden H, Berns A. Identification of cooperating oncogenes in E mu-myc transgenic mice by provirus tagging. Cell. 1991;65(5):737-752.

19. Valk-Lingbeek ME, Bruggeman SW, van Lohuizen M. Stem cells and cancer; the polycomb connection. Cell. 2004;118(4):409-418. 
20. Voncken JW, et al. MAPKAP kinase $3 p K$ phosphorylates and regulates chromatin association of the polycomb group protein Bmi1. J Biol Chem. 2005; 280(7):5178-5187.

21. Voncken JW, Schweizer D, Aagaard L, Sattler L, Jantsch MF, van Lohuizen M. Chromatin-association of the Polycomb group protein BMI1 is cell cycle-regulated and correlates with its phosphorylation status. J Cell Sci. 1999;112(pt 24):4627-4639.

22. Choudhary C, et al. Mislocalized activation of oncogenic RTKs switches downstream signaling outcomes. Mol Cell. 2009;36(2):326-339.

23. Christensen GL, et al. Quantitative phosphoproteomics dissection of seven-transmembrane receptor signaling using full and biased agonists. Mol Cell Proteomics. 2010;9(7):1540-1553.

24. Malik R, Lenobel R, Santamaria A, Ries A, Nigg EA, Korner R. Quantitative analysis of the human spindle phosphoproteome at distinct mitotic stages. J Proteome Res. 2009;8(10):4553-4563.

25. Olsen JV, et al. Global, in vivo, and site-specific phosphorylation dynamics in signaling networks. Cell. 2006;127(3):635-648.

26. Olsen JV, et al. Quantitative phosphoproteomics reveals widespread full phosphorylation site occupancy during mitosis. Sci Signal. 2010;3(104):ra3.

27. Pan C, Gnad F, Olsen JV, Mann M. Quantitative phosphoproteome analysis of a mouse liver cell line reveals specificity of phosphatase inhibitors. Proteomics. 2008;8(21):4534-4546.

28 . Rigbolt KT, et al. System-wide temporal characterization of the proteome and phosphoproteome of human embryonic stem cell differentiation. Sci Signal. 2011;4(164):rs3.

29. Weintz G, et al. The phosphoproteome of tolllike receptor-activated macrophages. Mol Syst Biol. 2010;6:371.

30. Zanivan S, et al. Solid tumor proteome and phosphoproteome analysis by high resolution mass spectrometry. J Proteome Res. 2008;7(12):5314-5326.

31. Jacobs JJ, Kieboom K, Marino S, DePinho RA, van Lohuizen $\mathrm{M}$. The oncogene and Polycombgroup gene bmi-1 regulates cell proliferation and senescence through the ink4a locus. Nature. 1999; 397(6715):164-168.

32. Gil J, Bernard D, Peters G. Role of polycomb group proteins in stem cell self-renewal and cancer. DNA Cell Biol. 2005;24(2):117-125.

33. Jacobs JJ, Scheijen B, Voncken JW, Kieboom K, Berns A, van Lohuizen M. Bmi-1 collaborates with c-Myc in tumorigenesis by inhibiting c-Mycinduced apoptosis via INK4a/ARF. Genes Dev. 1999; 13(20):2678-2690.

34. Bruggeman SWM, et al. Bmi1 controls tumor development in an Ink4a/Arf-independent manner in a mouse model for glioma. Cancer Cell. 2007; 12(4):328-341.

35. Xu CR, et al. Bmi1 functions as an oncogene independent of Ink4A/Arf repression in hepatic carcinogenesis. Mol Cancer Res. 2009;7(12):1937-1945.

36. Wu X, et al. Generation of a prostate epithelial cell-specific Cre transgenic mouse model for tissue-specific gene ablation. Mech Dev. 2001; 101(1-2):61-69.
37. Datta S, et al. Bmi-1 cooperates with H-Ras to transform human mammary epithelial cells via dysregulation of multiple growth-regulatory pathways. Cancer Res. 2007;67(21):10286-10295.

38. Guo W-J, et al. Mel-18 acts as a tumor suppressor by repressing Bmi-1 expression and down-regulating Akt activity in breast cancer cells. Cancer Res. 2007; 67(11):5083-5089.

39. Song L-B, et al. The polycomb group protein Bmi-1 represses the tumor suppressor PTEN and induces epithelial-mesenchymal transition in human nasopharyngeal epithelial cells. JClin Invest. 2009;119(12):3626-3636

40. Trotman LC, et al. Pten dose dictates cancer progression in the prostate. PLoS Biol. 2003;1(3):E59.

41. Elderkin S, et al. A phosphorylated form of Mel-18 targets the Ring $1 \mathrm{~B}$ histone $\mathrm{H} 2 \mathrm{~A}$ ubiquitin ligase to chromatin. Mol Cell. 2007;28(1):107-120.

42. Liu J, et al. Bmi1 regulates mitochondrial function and the DNA damage response pathway. Nature. 2009; 459(7245):387-392

43. Facchino S, Abdouh M, Chatoo W, Bernier G. BMI1 confers radioresistance to normal and cancerous neural stem cells through recruitment of the DNA damage response machinery. J Neurosci. 2010; 30(30):10096-10111.

44. Ismail IH, Andrin C, McDonald D, Hendzel MJ. BMI1-mediated histone ubiquitylation promotes DNA double-strand break repair. J Cell Biol. 2010; 191(1):45-60.

45. Ginjala V, et al. BMI1 is recruited to DNA breaks and contributes to DNA damage-induced H2A ubiquitination and repair. Mol Cell Biol. 2011; 31(10):1972-1982.

46. Fraser M, Harding SM, Zhao H, Coackley C, Durocher D, Bristow RG. MRE11 promotes AKT phosphorylation in direct response to DNA doublestrand breaks. Cell Cycle. 2011;10(13):2218-2232.

47. Nakanishi K, et al. Human Fanconi anemia monoubiquitination pathway promotes homologous DNA repair. Proc Natl Acad Sci U S A. 2005 102(4):1110-1115.

48. Berger R, et al. Androgen-induced differentiation and tumorigenicity of human prostate epithelial cells. Cancer Res. 2004;64(24):8867-8875.

49. Adams JM, et al. The c-myc oncogene driven by immunoglobulin enhancers induces lymphoid malignancy in transgenic mice. Nature. 1985 ; 318(6046):533-538

50. Shappell SB, et al. Prostate pathology of genetically engineered mice: definitions and classification. The consensus report from the Bar Harbor meeting of the Mouse Models of Human Cancer Consortium Prostate Pathology Committee. Cancer Res. 2004;64(6):2270-2305.

51 . Min J, et al. An oncogene-tumor suppressor cascade drives metastatic prostate cancer by coordinately activating Ras and nuclear factor-kappaB. Nat Med. 2010;16(3):286-294.

52. Dubrovska A, et al. The role of PTEN/Akt/PI3K signaling in the maintenance and viability of prostate cancer stem-like cell populations. Proc Natl Acad Sci US A. 2009;106(1):268-273.

53. Lukacs RU, Memarzadeh S, Wu H, Witte ON. Bmi-1 is a crucial regulator of prostate stem cell selfrenewal and malignant transformation. Cell Stem Cell. 2010;7(6):682-693

54. Niessen HE, Demmers JA, Voncken JW. Talking to chromatin: post-translational modulation of polycomb group function. Epigenetics Chromatin. 2009;2(1):10

55. Chou DM, et al. A chromatin localization screen reveals poly (ADP ribose)-regulated recruitment of the repressive polycomb and NuRD complexes to sites of DNA damage. Proc Natl Acad Sci U S A. 2010;107(43):18475-18480.

56. Richardson C, Stark JM, Ommundsen M, Jasin M. Rad51 overexpression promotes alternative doublestrand break repair pathways and genome instability. Oncogene. 2004;23(2):546-553.

57. Tutt A, et al. Mutation in Brca2 stimulates errorprone homology-directed repair of DNA doublestrand breaks occurring between repeated sequences. EMBO J. 2001;20(17):4704-4716.

58. Liu G, et al. Chromosome stability, in the absence of apoptosis, is critical for suppression of tumorigenesis in Trp53 mutant mice. Nat Genet. 2004; 36(1):63-68.

59. Kao SY, Lemoine FJ, Marriott SJ. Suppression of DNA repair by human $T$ cell leukemia virus type 1 Tax is rescued by a functional $\mathrm{p} 53$ signaling pathway. J Biol Chem. 2000;275(46):35926-35931.

60. Tran $\mathrm{H}$, et al. DNA repair pathway stimulated by the forkhead transcription factor FOXO3a through the Gadd45 protein. Science. 2002;296(5567):530-534.

61 . Toulany M, et al. Targeting of AKT1 enhances radiation toxicity of human tumor cells by inhibiting DNA-PKcs-dependent DNA double-strand break repair. Mol Cancer Ther. 2008;7(7):1772-1781

62. Deng $\mathrm{R}$, et al. PKB/Akt promotes DSB repair in cancer cells through upregulating Mre11 expression following ionizing radiation. Oncogene. 2011; 30(8):944-955.

63. Xu N, Hegarat N, Black EJ, Scott MT, Hochegger $\mathrm{H}$, Gillespie DA. Akt/PKB suppresses DNA damage processing and checkpoint activation in late G2. J Cell Biol. 2010;190(3):297-305.

64. Chen Z, et al. Crucial role of p53-dependent cellular senescence in suppression of Pten-deficient tumorigenesis. Nature. 2005;436(7051):725-730.

65. Chen Z, et al. Differential p53-independent outcomes of p19(Arf) loss in oncogenesis. Sci Signal. 2009;2(84):ra44.

66. You MJ, et al. Genetic analysis of Pten and Ink4a/Arf interactions in the suppression of tumorigenesis in mice. Proc Natl Acad Sci U S A. 2002;99(3):1455-1460.

67. Fan $\mathrm{C}$, et al. Bmi1 promotes prostate tumorigenesis via inhibiting p16(INK4A) and p14(ARF) expression. Biochim Biophys Acta. 2008;1782(11):642-648.

68. Ma X, et al. Targeted biallelic inactivation of Pten in the mouse prostate leads to prostate cancer accompanied by increased epithelial cell proliferation but not by reduced apoptosis. Cancer Res. 2005; 65(13):5730-5739.

69. Bric A, et al. Functional identification of tumorsuppressor genes through an in vivo RNA interference screen in a mouse lymphoma model. Cancer Cell. 2009;16(4):324-335. 